\title{
Stratified drought analysis using a stochastic ensemble of simulated and in- situ soil moisture observations
}

\author{
Vinit Sehgal \\ Department of Biological Systems Engineering \\ Virginia Polytechnic Institute and State University, Virginia, USA, 24060 \\ Venkataramana Sridhar* \\ Department of Biological Systems Engineering \\ Virginia Polytechnic Institute and State University, Virginia, USA, 24060 \\ Email: vsri@vt.edu; \\ *Corresponding Author \\ Aditya Tyagi \\ CH2M HILL, Austin, Texas, USA 78759
}




\section{Abstract}

This study proposes a multi- wavelet Bayesian ensemble of two Land Surface Models (LSMs) using in- situ observations for accurate estimation of soil moisture for Contiguous United States (CONUS). In the absence of a continuous, accurate in-situ soil moisture dataset at high spatial resolution, an ensemble of Noah and Mosaic LSMs is derived by performing a Bayesian Model Averaging (BMA) of several wavelet-based multi- resolution regression models (WR) of the simulated soil moisture from the LSMs and in-situ volumetric soil moisture dataset obtained from the U.S. Climate Reference Network (USCRN) field stations. This provides a proxy to the $i n$ - situ soil moisture dataset at $1 / 8^{\text {th }}$ degree spatial resolution called Hybrid Soil Moisture (HSM) for three soil layers $(1-10 \mathrm{~cm}, 10-40 \mathrm{~cm}$ and 40- $100 \mathrm{~cm})$ for the CONUS. The derived HSM is used further to study the layer- wise response of soil moisture to drought, highlighting the necessity of the ensemble approach and soil profile perspective for drought analysis. A correlation analysis between HSM, the long-term (PDSI, PHDI, SPI-9, SPI-12 and SPI-24) and the short-term (Palmer Z index, SPI-1 and SPI-6) drought indices is carried out for the nine climate regions of the U.S. indicating a higher sensitivity of soil moisture to drought conditions for the Southern U.S. Furthermore, a layerwise soil moisture percentile approach is proposed and applied for drought reconstruction in CONUS with a focus on the Southern U.S. for the year 2011.

Keywords: Soil Moisture, Land Surface Models, Drought, Wavelet analysis, Bayesian model averaging, Ensemble, NLDAS- v2 


\section{Introduction}

Drought and its impacts are not only incremental and a factor in long-term and cumulative environmental changes, but also comes into existence as a slow-onset or creeping event (Pulwarty and Sivakumar, 2014). Because it is difficult to establish a generally acceptable definition of drought for use in various water sectors, four types of droughts are defined as meteorological, agricultural, hydrological, and socioeconomic drought (Mozny et al., 2012; Wilhite and Glantz, 1985). Due to the slow onset and recovery, determining the beginning and end of a particular drought period is often difficult (Iglesias et al., 2009). In the wake of climate change, the likelihood of occurrence of extreme drought events with greater severity in the future in many regions of the globe is expected to rise significantly (Dai, 2011; Kundzewicz et al., 2010; Peterson et al., 2012; Trenberth and Fasullo, 2012). The areal extent of droughts increased more than 50\% during the last century over the globe, while changes in the areal extent of wet regions were relatively small (Trenberth et al., 2004). Hence, great emphasis has been laid out worldwide on the drought strategies, with a specific focus on comprehensive drought monitoring (Hayes et al., 2011).

Many studies have established the usefulness of soil moisture anomalies for characterizing drought onset and demise (Hunt et al., 2009). Soil moisture modulates the atmospheric surface energy balance and hence has a significant impact on the vertical distribution of turbulent heat fluxes, as well as the boundary layer structure (Alapaty et al., 1997). Soil moisture is a pivotal element in the hydrological process of converting precipitation into runoff and groundwater storage. Mozny et al. (2012) used in-situ soil moisture dataset for drought monitoring in the Czech Republic using a Soil Moisture Index (SMI) and found soil moisture based drought index to be useful in rapid-onset drought monitoring. Ford et al. (2015) used in-situ soil moisture observations for developing a flash drought early warning framework for Oklahoma. Sims and Raman (2002) established strong 
inter-relationship of two drought indices: Palmer Drought Severity Index (PDSI) values and Standardized Precipitation Index (SPI) with soil moisture for North Carolina. Lakshmi et al. (2004) showed that the deep layer soil moisture anomaly is a good drought indicator and has a higher memory than Palmer Drought Severity Index (PDSI).

To understand the interrelationship between soil moisture and drought at a large spatial scale, long-term data of soil moisture is required. However, in most regions of the world, the lack of spatially extensive soil moisture monitoring networks precludes the inclusion of soil moisture in drought monitoring systems (Ford et al., 2015). While some hydrological variables like precipitation, streamflow are more frequently recorded and reported, similar long-term soil moisture observations are scarce. There has been an extensive application of near-surface soil moisture information obtained through remote sensing in agriculture and meteorology. However, in most applications the magnitudes of modeled, in situ, and remotely sensed soil moisture often do not correlate well without any posterior bias correction (Narayan and Lakshmi, 2008; Sridhar et al., 2013). Also, near surface soil moisture in not sufficient to comprehensively assess drought severity. Hence, the use of simulated soil moisture from land surface models (LSMs) has become popular and has been applied to several studies to understand the dynamics and impact of drought. With the advancements in computational power, it is now feasible to generate operational land surface hydrological data products over large scales using telemetry, remote sensing based dataset and modeled meteorological data from atmospheric forecast models (Mitchell et al., 2004b). Though, the lack of high-resolution data to perform any physically based distributed hydrological modeling remains a limitation to researchers (Sridhar and Hubbard, 2009).

Previously, Sridhar et al. (2008) used simulated soil moisture using a hydrologic model developed by Robinson and Hubbard (1990) across different land-use conditions in Nebraska, US, to develop a Soil Moisture Index (SMI) for quantifying agricultural drought. 
The results were compared with the SMI derived from in-situ soil moisture. It was concluded that developing SMI using a simulation model-based approach should be considered pragmatic considering the difficulty in establishing a soil moisture observation-based drought index over large areas on a continuous long term. Sheffield et al. (2004a) used the Variable Infiltration Capacity (VIC) model to derive a model-based drought index and evaluated its agreement with the PDSI. Zhang et al. (2016) applied Australian Community Atmosphere Biosphere Land Exchange model (CABLE) LSMs to predict near-real-time drought in China. Yuan et al. (2013) used the VIC model for obtaining simulated soil moisture for probabilistic seasonal forecasting of droughts in Africa. Xia et al. (2014) used the North American Land Data Assimilation System (NLDAS) (Mitchell et al., 2004a) dataset to derive Objective Blended NLDAS Drought Index (OBNDI) for Conterminous United States (CONUS). Wang et al. (2009) developed a multimodal ensemble of several LSMs for reconstruction of drought over the U.S. AghaKouchak (2014) used precipitation and soil moisture dataset from NASA Modern-Era Retrospective analysis for Research and Applications (MERRA) for probabilistic drought forecasting using a standardized soil moisture index. Sheffield et al. (2014) used the VIC model to derive soil moisture for developing a drought monitoring and forecasting framework in Sub- Sahara region of Africa. Tang and Piechota (2009) used soil moisture obtained from the VIC model for spatial- temporal evaluation of drought variability in the Upper Colorado River Basin. Mo (2008) used Noah and VIC models from NLDAS in order to estimate soil moisture percentiles and runoff indices for drought analysis over U.S. AghaKouchak (2015) proposed a multivariate approach integrating soil moisture percentiles and precipitation for a persistence based drought prediction for East Africa. Soil moisture anomalies from NLDAS were used in assessing the evolution of soil moisture and vegetation conditions in extreme flash drought in Otkin et al. (2016). Wang et al. (2011) used an ensemble of four physically- based hydrology models to obtain soil moisture percentiles 
which were used to estimate the severity of agricultural drought. Sohrabi et al. (2015) developed a Soil Moisture Drought Index (SODI) to characterize droughts.

It is therefore evident the scientific community is interested in exploring the application of soil moisture in drought analysis. This study points the importance of differential response of soil profile soil moisture to drought. This insight augments the current understanding of soil moisture to drought correlation. This adds to the existing literature by proposing a layer- wise soil moisture percentile based drought reconstruction approach by using shallow soil layers for short- term, low-intensity drought reconstruction and deeper soil layers for capturing long-term and severe droughts, respectively. The study of the interrelationship between droughts and soil moisture at a large spatial scale is restricted to the use of several hydrological models which are themselves known to possess a high degree of uncertainties and biases (Mo et al., 2012). Although LSM-derived soil moisture shows encouraging consistency in the depiction of large-scale drought events, the development of a drought index based on soil moisture at a smaller scale appears to differ considerably across models(Xia et al., 2014). This is partly due to discrepancies in simulated soil moisture as well as sub-grid scale variability. Some recent studies have attempted to provide a stochastic approach to estimate in-situ soil moisture using hydrological models (Kim et al., 2015) but do not address the non- stationarity and multi-scale nature of the underlying process. This study utilizes an ensemble of Noah and Mosaic Land Surface Models (LSMs), obtained using a wavelet- based regression coupled with a Bayesian Model Averaging (BMA) approach (termed as WBR in this study) to simulate and evaluate soil moisture and its correlation with drought indices. The proposed wavelet-based multi-resolution modeling addresses to the nonstationarity and multiscale variability of soil moisture dataset by modeling the inputs in their sub- time series domain at multiple time- frequency resolutions. BMA is used to counter the subjectivity in the choice of mother wavelet function for time series decomposition into its 
sub- time series and uncertainty related to the ability of the selected wavelet to satisfactorily represent the process dynamics at the chosen level of time- frequency resolution.

The objectives of this study are as follows:

a) To develop an adjusted, high-resolution soil moisture dataset for carrying out drought sensitivity and reconstruction analysis for Contiguous United State (CONUS) using the proposed multi- resolution regression of Noah and Mosaic LSMs against in-situ soil moisture dataset obtained from U.S. Climate reference network (USCRN) stations.

b) To perform a layer- wise correlation analysis to understand the response of soil moisture to drought indices (DIs) representing short term (Palmer Z index, SPI-1 and SPI-6) and long term (PDSI, PHDI, SPI-9, SPI-12 and SPI-24) predictability of drought.

c) To highlight the difference in the spatial and inter- layer patterns in the persistence of the soil moisture pertaining to different soil layers, thus establishing a need for multilayer perspective in drought analysis and reconstruction using soil moisture.

d) To propose a new layer- wise drought reconstruction approach and apply to recent drought occurrences, for capturing drought in the Southern US (from April 2011December 2011) as a case study.

Section 2 of the paper provides a short description of the dataset used in this study. An overview of the Wavelet analysis and Bayesian Model Averaging is provided in Section 3. Development of the WBR models is explained in Section 4. Results are provided in Section 5 whereas Section 6 provides a detailed discussion on the observed results. The paper is concluded in Section 7.

\section{Data Description}




\subsection{In-situ soil moisture dataset:}

In-situ soil moisture observations for this study is obtained from the US Climate reference network (USCRN) (Bell et al., 2013) database. USCRN is a network of climate monitoring stations operated by the National Oceanic and Atmospheric Administration (NOAA). The soil moisture observations are available for five standard soil depths i.e. $5 \mathrm{~cm}$, $10 \mathrm{~cm}, 20 \mathrm{~cm}, 50 \mathrm{~cm}$ and $100 \mathrm{~cm}$ at daily and hourly time step (also sub-hourly time step for $5 \mathrm{~cm}$ layer). Currently, there are 114 stations operating in the continental United States out of which 87 stations contain soil moisture observations for all five layers. Furthermore, to facilitate the inter-comparison of dataset available from all layers, only the stations with equal length of dataset available for all layers are chosen.

Missing values in the dataset were filled using a penalized least square regression based on discrete cosine transform (DCT-PLS) algorithm proposed by Garcia (2010) and Wang et al. (2012). The PLS regression is a thin-plate spline smoother for generally onedimensional data array, which trades off fidelity to the data versus roughness of the mean function and thus expresses the data in terms of a sum of cosine functions oscillating at different frequencies (Wang et al., 2012). This algorithm is successfully employed by several other recent studies in order to address the issue of missing temporal data points in soil moisture (Dorigo et al., 2013; Su et al., 2016) , coastal ocean surface currents (Fredj et al., 2016), remotely sensed solar radiation estimated (Chen et al., 2014) etc. However, as a high number of missing values may lead to higher degree of uncertainty in the analysis, the stations with missing values greater than $15 \%$ of the total available dataset from the stations for any layer, are deemed to be unfit for this study.

A total of 48 stations meet all the aforementioned criteria of data availability for all layers and maximum missing values (Fig. 1(a)) and are termed as control stations in this 
study. In-situ soil moisture observations only from these control stations are used for building and validating the models which will be described later in this paper. Fig. 2 provides a plot for the observed soil moisture time series with gaps filled using the DCT- PLS algorithm for the stations with maximum number of missing values (below 15\% threshold) for each sensor depth in order to establish the effectiveness of the gap-filling algorithm for this study.

\subsection{Simulated volumetric soil moisture dataset from Noah and Mosaic LSMs:}

Simulated soil moisture dataset for this study is obtained from the North America Land Data Assimilation System Phase 2 (NLDAS-2) products. Simulated volumetric soil moisture variables from two land surface models namely Noah and Mosaic used in this study are available at a resolution of $1 / 8$ th of a degree for an extent of $25^{\circ} \mathrm{N}$ to $53^{\circ} \mathrm{N}$ latitude and $125^{\circ}$ W to $67^{\circ} \mathrm{W}$ Longitude.

The Mosaic Land Surface Model (LSM) developed by Koster and Suarez (1994) and implemented by Koster and Suarez (1996) as a tile approach to account the sub-grid vegetation variability. Each vegetation tile carries its own energy, water balance, soil moisture, and temperature. Each tile has three soil layers, first two of which are in the root zone. Noah model (Xia et al., 2012) was developed as the land component of the NOAANational Centers for Environmental Prediction (NCEP) mesoscale Eta model (Betts et al. (1997); Chen et al. (1997); Ek et al. (2003) ) and serves as the land component in the evolving Weather Research and Forecasting (WRF) regional atmospheric model, the Climate Forecast System (CFS), and the Global Forecast System (GFS). The model simulates the soil freeze-thaw process and its impact on soil heating/cooling and transpiration, following (Koren et al., 1999). Unlike Mosaic model, Noah has four soil layers, first three of which form the root zone in non-forested regions, and the fourth layer is included in forested regions (Mitchell et al., 2004a). 


\subsection{Drought Indices (DIs)}

Eight drought indices namely Palmer Drought Severity Index (PDSI), Palmer Hydrological Drought Index (PHDI), Palmer Z Index (ZNDX), Standardized Precipitation Index (SPI) for 1, 6, 9, 12 and 24 months are chosen for analysis in this study. The indices have been selected to facilitate a representation of a diverse set of drought scenarios. While indices like PHDI, PDSI, SPI-12, and SPI-24 relate to long-term droughts, ZNDX, SPI-1 and SPI-6 indicate relatively short- term drought predictability. While PDSI accounts for drought-inducing circulation patterns, PHDI is used to quantify hydrological effects of drought. PDSI, PHDI, and ZNDX are water balance indices (consider water supply, demand and loss through precipitation, evapotranspiration and runoff respectively), the Standardized Precipitation Index (SPI) is a probability index and relates to precipitation only. Monthly Palmer Drought Indices (PDSI, PHDI, and ZNDX) and SPI are obtained from the NOAA's National Climatic Data Center (NCDC-NOAA) repository for 344 climatic divisions in CONUS. For a given month, each point in a climatic division is assumed to have the same value for a drought index. Detailed information on the difference between these drought indices can be obtained from Mishra and Singh (2010).

Description of the layer thickness for Noah and Mosaic; and sensor location for the in-situ observations at USCRN sites is provided in Table 1. As the observed USCRN soil moisture is available for only up to first $100 \mathrm{~cm}$ of depth, the dataset for this study uses simulated soil moisture for three soil layers i.e. $0-10 \mathrm{~cm}, 10-40 \mathrm{~cm}$ and $40-100 \mathrm{~cm}$. Since the soil layers for USCRN data are different from those of Noah and Mosaic, a linear interpolation has been applied to obtain values at a depth of $5 \mathrm{~cm}, 25$ and $75 \mathrm{~cm}$ for an accurate layer to layer comparison. Soil moisture data from Noah, Mosaic and USCRN is converted to monthly time step for compatibility with the drought indices. 
Temporal length of the USCRN data varies from station to station based on installation, time for quality control and testing and operation of the soil moisture sensors. Broadly, the dataset from USCRN stations starts from Jan 2009 and goes up to August 2015. Equivalent lengths of the datasets from Noah, Mosaic have been obtained from their respective grids for the purpose of model development and validation. For the purpose of correlation and persistence analysis, and drought reconstruction, the data from Noah and Mosaic models for all three soil layers i.e. $0-10 \mathrm{~cm}, 10-40 \mathrm{~cm}$ and $40-100 \mathrm{~cm}$, and, the drought indices, are obtained for a period of Jan 1979 through Aug 2015.

\section{Brief overview of the mathematical techniques}

\subsection{Discrete Wavelet Transform (DWT):}

Natural processes are non-stationary and work at different spatial- temporal scales. The wavelet transform is a tool to obtain convolution of a signal or a time series against a particular instance of a wavelet corresponding to various time scales and positions. It breaks up a time series into shifted and scaled versions of wavelets, which are waveforms of effectively limited duration and zero mean, along the full signal in such a way that higher frequency wavelets will be very narrow and lower frequency wavelets will be wide. At each step, a generated wavelet coefficient measures the correlation of the wavelet to the signal in each section. This capability of the wavelet transform to focus on short time intervals for gross features and long intervals for local features makes it well suited for approximating data with sharp changes and discontinuities (Sahay and Sehgal, 2014).

Discrete wavelet transform (DWT) enables to achieve the time-frequency localization and multi-scale resolution of a signal by focusing and zooming around the neighborhood of one's choice (Mallat, 1999) for discrete time series, and a time series is decomposed into Approximation (A) and Detail (D1, D2, D3, etc.) sub-time series or Discrete Wavelet 
Components (DWCs) following a number of successive filtering steps . The Approximation components represent the coarse features of a time series which are obtained by correlating stretched version (low-frequency and high-scale) of a wavelet $\psi(n)$ with the original time series. The detail components signify rapidly changing features of time series and are obtained by correlating compressed wavelet (high-frequency and low-scale) with the original time series.

For a discrete time series $x_{i}$, DWT is defined as

$$
A_{m, n}=2^{-m / 2} \sum_{i=0}^{N-1} x_{i} \psi\left(2^{-m} i-n\right)
$$

Where $A_{m, n}$ is the wavelet coefficient for the discrete wavelet of scale $a=2^{m}, b=2^{m} n, m$ and $n$ are positive integer variable and $N$ is the data length which is equal to $2 M$. This gives the ranges of $\mathrm{m}$ and $\mathrm{n}$ as $0<n<2^{M-m}-1$ and $1<m<M$, respectively.

The original time series may be constructed using the Inverse Discrete Wavelet Transform as

$$
x_{i}=A+\sum_{m=1}^{M} \sum_{n=0}^{2^{M-m}-1} A_{m, n} 2^{-m / 2} \psi\left(2^{-m} i-n\right)
$$

Or, in simple terms,

$$
x_{i}=A(t)+\sum_{m=1}^{M} D_{m}(t)
$$

Where $A(t)$ is called approximation sub-time series at level $M$ and $D_{m}(t)$ are details sub-time series at levels $m=1,2, \ldots M$ 
Non-stationarity in the data such as trends and seasonal variations significantly affect modeling of soil moisture and hence may causes poor predictability in practical applications. Wavelet transforms, by analyzing the time series data in both time and frequency domains, give considerable information about the physical structure of the data which is useful to analyze time series that contain nonstationary power at many different frequencies (Daubechies, 1990). In the recent past, wavelet analysis has emerged as an attractive technique to deal with non-stationarity and multiscale processes. Wavelets have been applied in areas of hydrological modeling and time-frequency characterization of natural processes (Smith et al. (1998); Chou and Wang (2002); Wang and Ding (2003); Dai et al. (2003)). Extensive literature is available on wavelet-based models for a diverse set of problems in hydrological modeling like monsoonal flood forecasting (Sehgal et al., 2014c), drought forecasting (Kim and Valdés, 2003), streamflow (Adamowski, 2008; Coulibaly and Burn, 2004; Maheswaran and Khosa, 2012b; Nanda et al., 2016), precipitation (Kim, 2004; Lu, 2002; Partal and Kişi, 2007), climatic downscaling (Sehgal et al., 2016), hydrologic regionalization (Agarwal et al., 2016a; Agarwal et al., 2016b) and understanding the coherence between different hydro-climatic variables at multiple time-frequency resolutions like soil moisture (Lakshmi et al., 2004; Tang and Piechota, 2009), El Nino and Southern Oscillation (Torrence and Webster, 1998; Torrence and Webster, 1999) etc.

\subsection{Bayesian Model Averaging (BMA)}

Selection of an appropriate wavelet function poses significant challenges and is governed largely by the problem at hand and some of the distinctive properties of the wavelet function such as its region of support, and the number of vanishing moments (Maheswaran and Khosa, 2012a). Some other recent studies have also highlighted the effect of vanishing moment of the mother wavelet on the accuracy of a wavelet-based time series model (Sehgal et al., 2014a). The region of support relates to the length span of a wavelet thus effecting its 
feature localization capability, whereas, the vanishing moments, determine the ability of a wavelet to suitably represent the polynomial behavior of a time series. Thus, it is safe to assume that the performance of a wavelet-based multi- resolution model with a given mother wavelet function is highly dependent on the data, and, is location specific.

The best performing wavelet-based model at a given location may not perform satisfactorily at another location due to the choice of a given mother wavelet. Also, each mother wavelet captures a physical process at different time - frequency resolution. This study deals with a large geographic area (CONUS) with several climate regions having peculiar regional/ local features. Hence, it is incorrect to assume that a single wavelet will be able to capture the physical processes with a satisfactory degree of accuracy for the chosen highest level of decomposition in the study. Mostly, a single best model approach is applied in statistical modeling where a single model is chosen from a set of available and viable models based on various performance indices to assess agreement or disagreement of the modeled with the observed dataset. However, there is a possibility that a model which is able to capture some unique features of the processes of interest better than other models is being discarded as the said model may not perform best overall. Additionally, there may be uncertainties associated with estimated parameters for each model. The single best model ignores this aspect and leads to an overconfident estimation of the modeled variable (Fragoso and Neto, 2015; Hoeting et al., 1999).

BMA is a statistical approach for post-processing ensemble estimates from multiple competing models (Leamer, 1978). BMA helps in reducing this overconfidence and uncertainty related to a given model by conditioning on a given set of models. BMA has also been widely used in hydrologic and climatic modeling by providing a multi-model ensemble of for hydrologic predictions and forecasts (Duan et al. (2007), Ajami et al. (2007), Raftery et al. (2005), effective soil moisture estimation (Kim et al., 2015), drought forecasting 
(Madadgar and Moradkhani, 2013), groundwater modeling (Rojas et al., 2008), addressing model uncertainty (Parrish et al., 2012). Rathinasamy et al. (2013) proposed a multi- model ensemble using different wavelets for multi-scale streamflow forecasting. Vrugt and Robinson (2007) proposed a multi-criteria formulation for post-processing of forecast ensembles and applied on surface temperature and sea level pressure dataset. These studies report that a multi-model combination approach using BMA provides better results than those obtained using the individual models and helps in countering uncertainty associated with parameter estimation of a single model.

This study applies BMA on the top ten best performing wavelet-based regression models (WR) developed in this study in order to generate a combination soil moisture from Noah and Mosaic for each layer. This method assumes that the probability of the observed soil moisture $S M_{o b s}(t)$ at time $t$ is given by a weighted sum over the probability distributions $g\left(W R^{f}(k, t)\right)$ from the $k$ different WR models

$P\left(S M_{o b s}(t)\right)=\sum_{k} w(k) g\left(W R^{f}(k, t)\right)$

The choice of a probability distribution for each individual WR model is assumed to be a normal distribution following similar assumption from some recent studies (Kim et al., 2015; Rathinasamy et al., 2013; Vrugt and Robinson, 2007) with variance $\sigma(k)$

$g\left(W R^{f}(k, t) \mid S M_{o b s}(t), \sigma(k)\right)=(1 / \sigma(k) \sqrt{2 \pi}) e^{\left(-\frac{\left(s M_{o b s}(t)-W R(k, t)\right)^{2}}{2 \sigma(k)^{2}}\right)}$

For a given set of historic observed and simulated soil moisture using the WR Models, the BMA algorithm is used to obtain the optimal values for $w(k)$ and $\sigma(k)$, such that the likelihood of the overall PDF (Eq. 2) is maximal using an iterative process by consecutively optimizing $w(k)$ and $\sigma(k)$. The iteration is initialized with an estimate for the 
weights $w(k)$ and $\sigma(k)$ for each individual model and estimates of matrix $z(k, t)$ using Eq. (3). $z(k, t)$ gives the probability that model $k$ is the best estimate for the soil moisture at time $t$

$z(k, t)=g\left(S M_{o b s}(t) \mid W R(k, t), \sigma(k)\right) / \sum_{k} g\left(S M_{o b s}(t) \mid W R_{f}(k, t), \sigma^{2}(k)\right)$

In the second step, an iterative algorithm estimates the weights $w(k)$ and variances $\sigma(k)$ of each of the model's $k$ based on the values of $z(k, s, t)$. . The weights are estimated as;

$w(k)=\frac{1}{n} \sum_{t} z(k, t)$

where $n$ is the number of observations in the training period $(t)$. The variance $\sigma(k)$ is estimated using

$\sigma^{2}(k)=\left(\sum_{t} z(t)\left(S M_{o b s}(t)-W R_{f}(k, t)\right)^{2}\right) / \sum_{t} z(k, t)$

The last two steps are alternated in this process in order to achieve a desired degree of convergence using Expectation-Maximization (EM) algorithm proposed by (Raftery et al., 2005). For detailed information on the Bayesian Model Algorithm applied in this study, the readers are referred to Fernandez et al. (2001a) and Fernandez et al. (2001b).

\section{Development of multi- resolution regression based Bayesian Model Averaging (WBR) models}

In order to address the need of an accurate in-situ soil moisture dataset at high spatial resolution, and to counter the uncertainty and bias in the soil moisture available from the two LSMs namely, Noah and Mosaic (obtained from NLDAS-v2) for this analysis , a statistical transformation of volumetric soil moisture provided by the two LSMs is carried out to obtain a Hybrid Soil Moisture (HSM) for the Conterminous United States (CONUS) for three soil layers i.e. $1-10 \mathrm{~cm}, 10-40 \mathrm{~cm}$ and $40-100 \mathrm{~cm}$ at a spatial resolution of $1 / 8^{\text {th }}$ degree. 


\subsection{Wavelet based multi- resolution regression models (WR models)}

The observed soil moisture dataset is obtained from the selected 48 control stations. The in-situ and simulated soil moisture dataset obtained from all control stations for USCRN, Noah and Mosaic is appended station-wise in order to obtain a single time series for each source. These time series are used as inputs to the statistical models.

The input time series are first standardized prior to the model development to reduce systematic biases in the mean and variance values of Noah/ Mosaic dataset. A multiresolution modeling approach is employed in this study where each wavelet sub- time series of the input data is modeled separately using regression-based framework for respective subtime series of the in-situ soil moisture dataset. Each model provides the respective sub-time series of the HSM which is later added to provide the HSM in original time - frequency domain. This modeling approach is called as the Wavelet-based multi- resolution regression (WR) in this study. Selection of suitable level of decomposition is important in a multiresolution modeling. A schematic of the framework for the WR models in provided in Fig. 3.

\subsubsection{Selection of suitable level of decomposition of input dataset for WR models}

Selection of resolution of time series decomposition using DWT is conducted by observing the wavelet spectrum of the observed soil moisture time series. Fig. 4 provides the wavelet spectrum of the observed soil moisture using Morlet wavelet. It can be observed that there are significant occurrences around the $9^{\text {th }}$ level of decomposition (256- 512 months) for all three layers. This can be attributed to the effect of El Niño Southern Oscillation (ENSO) and Pacific Decadal Oscillation (PDO) which are two important oceanic-atmospheric indices having a strong influence on the climate variability in large parts of the US (Barnett et al., 1999; Beebee and Manga, 2004). It can be observed from Fig. 4 that apart from a consistent annual variability, the soil moisture for all three layers consistently displays a decadal 
variability pertaining to a $256-512$ month period. Hence, the dataset from the three sources is decomposed up to nine time- frequency resolutions and modeled separately to obtain the estimated DWC of the HSM at the respective time- frequency resolution.

\subsubsection{Selection of wavelets for WR models}

Maheswaran and Khosa (2012a) and Sehgal et al. (2014a) showed that wavelets with high support width and higher vanishing moments are better suited to capture the dynamics of a time series at multiple time-frequency resolutions. "daubechies" $(d b)$ wavelets are used in this study which provide a high number of vanishing moments for a given support width (Sehgal et al., 2014b). A WR( $(i)$ is developed using $d b(i)$ wavelet for obtaining the DWCs of the input dataset for the models where $i$ represent the vanishing moment of the employed mother wavelet for the WR model and ranges from 1 through 45.

\subsection{Bayesian Model Averaging of Wavelet based multi- resolution regression Models (WBR} models)

BMA is applied to obtain an overall probability distribution function (PDF) of the volumetric soil moisture by taking a weighted average of outputs from top ten individual WR models using standardized Noah and Mosaic soil moisture inputs, each employing a different wavelet as mother functions. Fig. 5 provides a schematic of the proposed modeling framework applied in this study. Top ten WR models are selected based on the model's correlation with the observed dataset. These models are then fed into the BMA framework to obtain weights for each WR model which are then used to obtain an ensemble of the top ten WR model by weighted averaging, providing the $\mathrm{WBR}_{\text {Hybrid }}$ output. The best performing wavelets obtained regression coefficients corresponding to each model run and the respective model weights are recorded in the form of a "Transformation matrix" for each layer as this 
matrix provide all necessary information to obtain the HSM from Noah and Mosaic dataset for each grid cell.

\section{Results}

\subsection{Comparison of Noah and Mosaic simulations with observed soil moisture dataset}

It is important to analyze the discrepancies in the model simulated soil moisture using Noah and Mosaic compared to the observed in- situ soil moisture to establish the necessity of the proposed ensemble approach. An analysis is carried out to understand the problem of over/ underestimation of soil moisture for each control station for the three soil layers. If the estimation of soil moisture using the LSMs is between $\pm 10 \%$ of the observed data value, the estimation is termed as satisfactory otherwise the soil moisture is either over/ under predicted. Fig. 6 provides the percentage of data points for each control station being over $(\mathrm{O}) /$ satisfactorily (S)/ or under (U) - predicted. It can be observed that for most of the stations, both Noah and Mosaic over-estimated the soil moisture. Overall, the overestimation is observed to increase with layer depth.

\subsection{Performance evaluation of developed models}

\subsubsection{Statistical indices for performance evaluation}

In order to evaluate the performance of developed models, the dataset for each control station is divided into a fraction of 3:1 where first three-quarters are used as a training dataset and the later quarter of the data for each control station is used in the validation process. In total, the training dataset consisted of 2204 data points whereas the validation dataset consisted of 727 values. In order to assess performance of the developed models, following error metrics are evaluated in this study:

(i) Normalized Root Mean Square Error (NRMSE) is Root Mean Square Error (RMSE)

normalized to a scale $[0,1]$. 
RMSE is expressed as:

$R M S E=\sqrt{\frac{1}{n} \sum_{i=1}^{n}\left(O_{i}-P_{i}\right)^{2}}$

Where $O_{i}$ and $P i$ are the observed and estimated SM time series and $n$ is the number of data points in the series. To facilitate easy comparison of model performances across stations and models, NRMSE is adopted in this study which is expressed as:

NRMSE $=$ RMSE/ Range

Where, Range is the difference between the maximum and the minimum value of the observed dataset.

(ii) Nash-Sutcliffe coefficient (E). It is expressed as:

$E=1-\frac{\sum_{i=1}^{n}(O i-P i)^{2}}{\sum_{i=1}^{n}(O i-\overline{O \imath})^{2}}$

Where $\overline{O_{l}}$ is the mean of the observed soil moisture.

(iii) Correlation coefficient (CC) is defined as:

$C C=\left(\frac{\sum_{i=0}^{n}(\mathrm{Oi}-\bar{O})(\mathrm{Pi}-\bar{P})}{\sqrt{\sum_{i=0}^{n}(\mathrm{Oi}-\bar{O})^{2}} \sqrt{\sum_{i=0}^{n}(\mathrm{Pi}-\bar{P})^{2}}}\right)$

Where $\bar{P} l$ is the mean of the estimated soil moisture.

\subsubsection{Performance evaluation}

Results of performance analysis for the total dataset (training + validation) is summarized in Table 2 ( $a, b$ and $c$ ) for layer 1, 2 and 3 respectively. In these tables, the performance of the top $10 \mathrm{WR}$ models is tabulated along with WBR ${ }_{\text {Hybrid }}$ models for the 
purpose of comparison. These tables also provide the model weights applied to the respective model output. The value in the parenthesis of the WR models provides the vanishing moment of the $d b$ wavelet selected as mother wavelet for the WR model. For example, $W R(7)$ signifies the WR model with $d b 7$ as the mother wavelet function. Fig. 7 provides the scatterplots of the observed dataset and HSM for the validation and total dataset. It can be observed from Fig. 7 that no systematic over- or under- estimation in the model outputs.

Table 3 provides the information about the performance of WBR Hybrid $_{\text {models for }}$ each layer for training and validation dataset. For the purpose of comparison, the performance of the best performing WR models for the respective layer is also included in Table 3. For validation data, the HSM (WBR ${ }_{\text {Hybrid }}$ output) provides a correlation of $0.78,0.75$ and 0.73 for layer 1, 2 and 3 respectively. The values of $E$ for the three layers for the validation dataset are $0.6,0.57$ and 0.53 whereas NRMSE is found to be $0.15,0.159,0.154$ for layer 1, 2 and 3 respectively. Respective values for the performance indices for the WR model outputs fall short of the WBR model outputs in performance for both training and validation dataset. The values for correlation, E and NRMSE for layer 1, 2 and 3 for the WR model outputs for validation dataset are $0.78,0.70$ and $0.71 ; 0.59,0.48$ and $0.50 ; 0.152,0.174$ and 0.160 respectively.

Different combinations of inputs and modeling approaches have been explored for their performance in estimating the observed soil moisture for the purpose of comparison with the proposed hybrid model of Noah and Mosaic using Wavelet regression and BMA

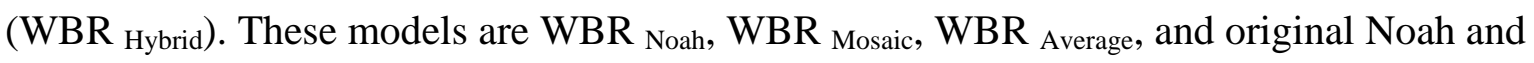
Mosaic dataset. A brief description of these models is provided in Table 4.

Table 5 summarizes the performance of the models developed for comparison with

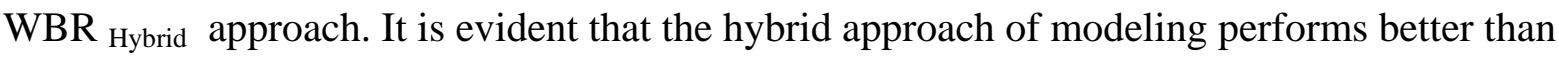


all other models. Also, it can be observed from Table 2, Table 3 and Table 5 that models perform comparatively better for layer 1 than layer 2 and 3 . This can be noticed by observing that the correlation of soil moisture inputs from Noah and Mosaic decreases from layer 1 to layer 3. As the input variables had less correlation with the observed dataset with increasing depth from the surface, the performance of models falls from layer 1 to layer 3 . However, it is noted that for each layer, the HSM has a higher correlation with the observed dataset than that of Noah/ Mosaic and observed data. It can be observed from Table 5 that WBR Hybrid models outperform the best performing models for all performance indices for layer 2 and 3 . For Layer 1, though WBR ${ }_{\text {Hybrid }}$ Outputs do not provide the best performance, however, the performance is very close to the best WR outputs.

Fig. 8 provides a comparison of the histograms obtained for the correlation of in- situ soil moisture from control stations with HSM, Noah, and Mosaic. It can be seen that while the overall correlation of the three datasets with the observed soil moisture falls with an increase in the layer depth, HSM consistently provides higher number of stations with a higher correlation than Noah and Mosaic for all three layers. In order to test whether HSM captures the multi- scale variability of the in- situ dataset, a comparison between the wavelet spectrums of the observed in-situ dataset, HSM, Noah and Mosaic dataset is provided in Fig. 9. It is evident from the figure that while the observed dataset has significance towards higher periods (due to decadal variability), Noah and Mosaic datasets are not able to reflect significance in this range. However, HSM effectively captures this variability and displays significance around 128 to 512 months similar to the observed dataset.

\subsubsection{Performance evaluation using remotely sensed dataset}

The developed HSM is validated further with the remotely sensed dataset available from the Soil Moisture Active and Passive (SMAP) mission. The dataset from SMAP is 
available only for the top soil layer $(\sim 5 \mathrm{~cm})$. Due to data availability issues, the validation is carried out for two months (June and July 2015). It can be observed from Fig. 10 that the HSM is sensitive able to counter the wet bias of the two LSMs and is relatively more sensitive to dry conditions which make it suitable for drought analysis.

\section{Drought analysis using HSM}

While the knowledge of past climatology is important for obtaining the soil moisture percentiles, the available in-situ dataset for this study is relatively short ( $<7$ years). This may influence the stability of the soil moisture percentiles developed for the purpose of drought reconstruction and/ or the study of persistence of soil moisture for CONUS, and, the correlation between soil moisture and the selected drought indices. Hence, the transformation matrix is applied to Noah and Mosaic dataset from Jan 1979 to Aug 2015 in order to obtain a data record of HSM for $\sim 37$ years. This dataset is used for further analysis described in this section.

\subsection{Layer-wise persistence of soil moisture}

A layer-wise analysis is conducted to study the persistence of the soil moisture for the CONUS to highlight the variation in the properties of the soil moisture for different soil layers. Persistence is a distinctive characteristic of soil moisture as it exhibits less variability relative to precipitation (Hao and AghaKouchak, 2014). Previously, Mo et al., 2012a illustrated the importance of soil moisture persistence in improving the drought forecasting skills. The persistence of the soil moisture time series is obtained in terms of the response time $\left(T_{o}\right)$ of the HSM for each grid cell. Higher persistence indicates longer memory of the soil moisture, hence delayed response to drought and vice-versa. Response time $\left(T_{o}\right)$ (Delworth and Manabe, 1988; Trenberth, 1984) is defined here as the lag time at which the 
autocorrelation of the time series of soil moisture has decayed to $1 / e \cdot T_{o}$ of a time-series can be calculated from the autocorrelation $R(i)$ at lag $i$ month for $i=1$ to 30 :

$T_{o}=1+2 \sum_{i=1}^{N}(1-i / N) R_{i}$ with $N=30$

Fig. 11 shows the spatial distribution of $T_{o}$ obtained from the soil moisture percentiles for the period Jan 2009 to Aug 2015 for CONUS. Further, the response time for each soil layer is averaged for the nine climate regions (as specified by Karl and Koss (1984) and shown in Fig. 1 (b)) of the US and is shown in Fig. 12.

It can be observed that the spatial patterns of $T_{o}$ changes drastically with increasing the layer depth. For Layer 1, the value of $T_{o}$ over the region that covers Texas, New Mexico, Oklahoma, Kansas, North and South Dakota with some regions of Georgia and South Carolina is observed to be around 4-6 months compared to Pennsylvania, New York, and Michigan in the East and Washington, Oregon, Idaho and some parts of California which show a higher $T_{o}$ of about 12- 15 months. For Layer 2, the value for $T_{o}$ decreases for major parts of Oklahoma, New Mexico, and Texas whereas for the east and northeastern states it increases to 15-16 months. For Layer 3, the spatial variations in the $T_{o}$ values show reductions due to overall alleviation in the $T_{o}$ values across the CONUS with some regions like Oklahoma, and some parts of Texas, North Carolina, and South Carolina showing low $T_{o}$ values. Overall, it can be observed from Fig. 11 and Fig. 12 that the $T_{o}$ for the southern part of the CONUS has lowest response time compared to other regions. This is in accordance with the work of Wang et al. (2009) and Mo (2008) who reported higher persistence of soil moisture across the U.S. Karl (1983) attributed the abnormally wet or dry weather in the Rocky Mountain (around Colorado, Idaho, Montana) and the High Plains states (around Nebraska, Kansas and South Dakota) for higher persistence in soil moisture than the states farther east or west. Wang et al. (2006) highlighted that factors pertaining to soil depth and 
soil characteristics like porosity, texture, vegetation of a soil are significantly related to the response time of a soil. Hence, it is evident that there is a great degree of variation in the soil moisture behavior across layers with respect to their persistence defined in terms of $T_{o}$.

\subsection{Layer-wise sensitivity of soil moisture to drought indices}

The transformation matrix for each layer contains the information about the top performing wavelets, the regression coefficients for each WR model and the respective model weights. These transformation matrices are applied over all grid cells of NOAH and MOSAIC to obtain HSM at $1 / 8^{\text {th }}$ degree resolution from Jan 1979 to Sep 2015 at monthly scale for all three layers. Once the dataset for all grid cells is obtained, the correlation between the HSM for all three layers with the eight DIs for their respective climate division is obtained for each grid. This exercise provides a spatial map of the inter-relationship between each of the eight DIs under study with the HSM for all three layers. Fig. 13 shows the spatial pattern of correlation of soil moisture with the DIs for CONUS. Furthermore, the average correlation of soil moisture with DIs for each climate region is summarized in Fig. 14.

The observable trends can be summarized in the following points: Regions like the west north central part of US around Texas and New Mexico, West south central (Kansas, Nebraska) and regions around Georgia, Alabama and South Carolina typically show a higher correlation with the DIs compared to other regions of respective layers. In contrast, the region around the mid-Atlantic US, New England and the Western US show very low correlation with the drought indices. It is also worth noting that these regions have significant low persistence in comparison with other regions for the soil moisture of a given layer. Hence, persistence is observed to have a significant impact on the response of soil moisture to drought. 
These trends can be attributed to the fact that the role of soil moisture in modulating precipitation generation is extremely high in the 'transition zones' between wet and dry climates, as evaporation becomes sensitive to soil moisture in these areas (Koster et al., 2004). The Great Plains can be characteristically identified with this phenomenon simply due to the fact that evaporation is greater than precipitation during the growing season, thus implying that the region predominantly serves as a source for moisture(Sridhar and Wedin, 2009).

\subsection{Effect of autocorrelation in time series on correlation analysis}

Significant autocorrelation in the soil moisture and DI time series may impact the correlation analysis (and thus, its inferences). In order to eliminate any significant autocorrelation and seasonal effects, the time series are pre- whitened prior to the correlation analysis. Prewhitening is carried out using ARIMA (Box and Jenkins, 1976) $(2,1,2)$ models to obtain the residuals for the time series of soil moisture and drought indices for each grid cell. An analysis is carried out to select the most suitable ARIMA model for the purpose of prewhitening (refer to the Supplementary material). 20 grid points are randomly selected across CONUS and ARIMA ( $p, 1, q)$ models are evaluated for the lowest AIC for different combinations of $\mathrm{p}$ (autoregressive component) and $\mathrm{q}$ (moving average component) where $\mathrm{p} \in$ $[0,1,2]$ and $\mathrm{q} \in[0,1,2]$. A sample analysis of correlation analysis using the pre- whitened soil moisture and DI time series is shown for Z-index and SPI-1 in Fig. 15. On comparing Fig. 14 and Fig. 15, it is evident that the spatial pattern of correlation between the two DIs and soil moisture for each layer is different when the pre- whitening of the time series is carried out prior to calculating the correlation between the two variables. However, the interpretation about the relative strength of the interrelationship from layer 1 through layer 3 isn't effected. Since the correlation strength is impacted by pre-whitening, while the patterns remain unchanged, it is generally recommended to do pre-whitening. However, care should be given 
to retain the physical relationship in the variable (in this case soil moisture) and its relationship with DIs. As the ultimate goal is to evaluate if soil moisture can be useful in predicting drought, correlation analysis need not done if the resultant data dilutes the nature of relationship. However, lack of information on soil moisture data for the managed and natural systems, a generalized approach to remove the temporal correlation across the entire domain constrained this study to use the data without pre-whitening. This assumption is consistent with some studies which utilize the autocorrelation in soil moisture as an important property in order to capture the perpetuation of drought conditions. Tang and Piechota (2009) and Lakshmi et al. (2004), based on the autocorrelative structure of soil moisture, conclude that the deep layer soil moisture anomaly possess strong inter-relationship with drought indicators like PDSI. Also, Oglesby et al. (2002) show that deep soil zone anomalies exert a more powerful, long-lasting effect than anomalies in the near-surface soil zone and hence, are more important in explaining interannual to decadal droughts evident in the historic and recent prehistoric records.

Hence upon comparing the results of correlation between the DIs and soil moisture for all three layers with and without pre- whitening of the input time series, and evaluating related literature, this study utilizes the correlation results of soil moisture and the DIs without pre-whitening.

\subsection{Layer-wise drought reconstruction for Southern U.S.}

Based on the correlation and sensitivity of drought indices for soil moisture, it is clear that each soil layer responds to drought in a different way. While top soil layer has comparatively low persistence, it is expected to respond quickly to impending drought conditions compared to the deeper layers. At the same time, the deeper soil layer will respond 
slowly to changing moisture conditions and hence will respond slowly to improving drought conditions.

Currently, soil moisture percentiles used in drought reconstruction provide an estimate of moisture percentiles for total column length of soil moisture (Luo and Wood, 2007; Wood, 2008) and does not account to this inter- layer variations in moisture loss. The USDM provides a comprehensive drought assessment of current drought conditions for the US and provides a useful reference for socio- economic and agricultural management purposes at a weekly time step (Wang et al., 2009). USDM drought assessment maps are popularly used for validating the drought assessment from various research products with that of an operational product. The USDM classification of drought severity using soil moisture percentile is given in Table 6. HSM (Jan 1979 through Aug 2015) is used to in determining the thresholds for each severity level, and all data used in drought severity determinations are considered with reference to their historical frequency of occurrence for the location and time of year in question in accordance with Svoboda et al. (2002).

\subsubsection{Layer-wise classification of soil moisture percentile to drought severity}

The average squared correlation coefficient (ASCC) (Abatzoglou et al., 2014) is employed to relate the soil moisture percentile with long/ short term drought. ASCC is obtained by averaging the squared correlation coefficient between the drought indices and soil moisture for the three layers obtained for all available data points. The ASCC values obtained from all grids across CONUS are normalized across layers to obtain the normalized average squared correlation coefficient (NASC) to facilitate a comparative assessment of the strength of the relationship between the DI and HSM across different layers as provided in Fig. 16 against the values obtained from the in-situ observations. The layer-wise sensitivity 
provides information of the relative sensitivity of a given drought index across layers from the two datasets.

From Fig. 16, it can be observed that the top soil layer (Layer 1) is more responsive to the short-term variations in moisture regime due to direct soil evaporation whereas Layer 3 reflects more on the long term moisture deviations and often display a lagged response to dry conditions. Layer 2 served as a link between the two layers in terms of transition of drought from low to high severity. This understanding is used while proposing a new soil moisture percentile classification to effectively represent drought severity for the Southern U.S. While Layer 1 is used to represent only the transient dry conditions (D0), Layer 3 is used to capture severe and long-term droughts (D2-D4). Layer 2 is used to represent the transition of drought severity from low to high (D0-D3). The effectiveness of the drought reconstruction approach is carried out against the USDM maps for the concurrent time. The layer-wise soil moisture percentiles and corresponding drought severity proposed in this study are provided in Table 7.

\subsubsection{Application of proposed multi-layer percentiles for drought reconstruction for} CONUS. (April- December 2011)

In the proposed approach, the drought classification is carried out for each layer using the classification provided in Table 7. Drought severity maps obtained from each layer are then overlaid with deeper layers getting higher priority than the shallower ones. To illustrate, if we assume the moisture percentile for a grid cell is observed between 5-2 percentile for all layers simultaneously, then the drought will be classified as a D3 drought (Extreme drought).

Drought reconstruction maps using the proposed multi-layer percentile approach for CONUS (with a focus on drought in the southern U.S.) for April- December 2011 is provided in Fig. 17. It can be observed that the soil moisture percentiles are able to respond in 
accordance with the drought conditions as depicted by the USDM maps. Consistent with the USDM approach, the percentiles used for the drought severity classification in this study are standardized for the time of the year, rather than for all times of the year at once. It is to be noted that though the threshold values for drought severity using the soil moisture percentiles do not correspond exactly to the appropriate soil type-based water holding capacities and their percentiles at all locations across the U.S., they provide a consistent and replicable standard for drought classification utilizing a variable easily understood by the general public. These objective inputs, and subjective adjustments based on local impacts and vulnerability result in a drought indicator based on a convergence of evidence that can be interpreted easily in terms of return periods (Svoboda et al., 2002).

A similar drought reconstruction is carried out using Noah and Mosaic dataset for the purpose of comparison (Fig. 18). Discrepancies between the drought maps reconstructed using Noah and Mosaic can be seen in the plots. While two models generally agree on severe droughts in the Southern U.S. for the study period, there remains significant dissimilarities in the models in terms of severity and the region under estimated severity conditions for other regions. A comparison of the area under various drought severity conditions represented by the reconstruction approach using HSM, Noah and Mosaic, and, the USDM for April through Dec- 2011 (months of most severe impact) is provided in Fig. 19. Fig. 20 provides a comparison between the time series of the eight drought indices and monthly soil moisture percentiles using HSM, Noah and Mosaic. It can be seen from Fig. 19 and Fig. 20 that there remain discrepancies in analysis based on Noah and Mosaic dataset, while HSM provides a reliable ensemble of the two models and is able to estimate in- situ soil moisture with greater accuracy, thus eliminating the subjectivity in choosing one model over another. 


\section{Discussion}

A layer- wise perspective is imperative to completely represent the water transport dynamics in soil column in the event of drought. While, the drier conditions in the near surface soil layer may indicate short-term meteorological drought it may not be a good measure of agricultural drought if the total root zone water storage is relatively wet. Deficit soil moisture storage in the total soil column may be indicative of hydrological drought but a single storm may replenish root zone soil moisture enough to relieve agricultural drought, yet hydrological drought could inflict the region over a season (Sheffield et al., 2004b). Deeper layers represent the water availability for planT uptake, aquifer recharge, and, baseflow to streams, whereas, the near- surface soil moisture indirectly indicates the dryness of the deposited plant litter which may indicate susceptibility to other natural hazards like wildfire, essentially, aggravating drought conditions. A detailed study on the dynamics of soil moisture can be found in D'Odorico et al. (2000).

It can be observed that the reconstructed maps are quick to respond to transient changes and usually predate the USDM maps in capturing the drought onset. This property can be attributed to the inclusion of moisture percentile information from shallow soil layers in representing flash droughts. Further applicability of the proposed approach for drought early forecasting with satisfactory skill- scores and lead time can be useful to effectively characterize emerging drought conditions over large areas. The proposed representation of drought severity is directly comparable to the USDM maps, which are popular benchmarks for drought analysis, making it easier for the policy makers in taking decisions based on evolving hydrometeorological conditions. While the climatology and dataset for the USDM and this study are not the same, the USDM involves many other factors like county-level information on drought, reservoir levels, snowpack and groundwater which may lead to different interpretation of drought at local scales. 
Timely identification of impending drought conditions is important from agricultural and water management perspectives. The two land surface models for this study namely Noah and Mosaic are available at near real- time through the NLDAS- v2 platform. Using the ensemble model proposed in this study, HSM can be generated in near- real time mode and hence carries the potential to be used in an operational drought monitoring setup. Also, soil moisture simulations from multiple physically based models can be derived using the seasonal forecasts from coupled ocean-atmosphere general circulation models (Wood et al., 2002) and a similar approach can be applied to obtain HSM and thus soil moisture percentiles for forecasting droughts with a lead time of several months.

However, the proposed approach has certain limitations due to the model design and limited availability of observed data. The proposed approach for drought reconstruction is developed in a time independent way, which means, that the drought severity is estimated for the current month and hence compromise on maintaining the continuity during month-tomonth transitions and doesn't account for the contribution of the additional soil moisture for hydrologic processes in the next time step.

Currently, this study uses in- situ soil moisture data from 48 observation stations available for a relatively short time duration (Jan 2009 through Aug 2015). This limited sampling could potentially impact the performance of the proposed approach. Although the issue of limited data availability is addressed, to some extent, using a generalized modeling approach consisting of multiple wavelets and BMA to capture the overall variability of the data across CONUS, a higher number of observation stations with longer data record will certainly add to the accuracy and confidence of the model. Recent efforts in soil moisture monitoring like North Atlantic Soil moisture database (Quiring et al., 2015), and advancements in remotely sensed soil moisture missions like SMAP (Brown et al., 2013), will be able to rectify this issue in coming years. It is also to be noted that the current drought 
reconstruction is performed at a monthly time step. Increasing the temporal resolution will add to the ability of the drought percentiles to capture short term changes.

\section{Conclusion}

Soil moisture is an important tool in understanding the onset, propagation, and recovery of droughts. However, as an alternative to a long-term, in- situ soil moisture data for drought analysis, this study develops an ensemble of Noah and Mosaic Land Surface Model estimated soil moisture and in- situ observations to obtain a combined dataset data called Hybrid Soil Moisture (HSM). The HSM is used to understand the response soil moisture from three layers $(0-10 \mathrm{~cm}, 10-40 \mathrm{~cm}$ and $40-100 \mathrm{~cm})$ to eight drought indicators representing long-term and short-term drought predictability. A stratified approach for reconstruction of droughts is proposed in this study and is used for drought reconstruction over Southern U.S. during a recent drought to demonstrate the effectiveness of the proposed approach in capturing the drought dynamics. This study can be summarized as follows:

- A statistical approach is employed to obtain a hybrid of two Land Surface Models for the CONUS using in- situ dataset to reduce bias and error in estimating the true state of soil moisture. A multi- resolution regression coupled with Bayesian Model Averaging is used for transforming the simulated soil moisture from the two LSMs into Hybrid Soil Moisture which is further used as a proxy to in- situ soil moisture for CONUS scale statistical analysis and drought reconstruction for Southern U.S.

- A layer- wise correlation analysis of the soil moisture with eight drought indices namely PDSI, PHDI, Z index, SPI- 1, 6, 9, 12 and 24 shows that shallow soil layers $(0-10 \mathrm{~cm}$ and $10-40 \mathrm{~cm})$ respond to short-term drought occurrences with 
higher correlation whereas deeper layer $(40-100 \mathrm{~cm})$ is more responsive to longterm droughts.

- The three soil layers under study differ significantly with respect to their Response Time, which significantly drives their characteristic response to drought. This strengthens the argument in favor of a layer- wise approach for drought analysis and reconstruction.

- A new drought classification based on stratified soil moisture percentiles is proposed and applied to the reconstruction of drought over CONUS with a focus on drought in the Southern U.S. for Apr- Dec 2011. The reconstructed drought maps show good correspondence to the USDM drought maps for the concurrent period. The reconstructed maps usually predate the USDM maps in representing low-intensity droughts which can be exploited further in flash drought studies. 


\section{References}

Abatzoglou, J.T., Barbero, R., Wolf, J.W., Holden, Z.A., 2014. Tracking interannual streamflow variability with drought indices in the US pacific northwest. Journal of Hydrometeorology, 15(5): 1900-1912.

Adamowski, J.F., 2008. River flow forecasting using wavelet and cross- wavelet transform models. Hydrological processes, 22(25): 4877-4891.

Agarwal, A., Maheswaran, R., Kurths, J., Khosa, R., 2016a. Wavelet Spectrum and SelfOrganizing Maps-Based Approach for Hydrologic Regionalization-a Case Study in the Western United States. Water Resources Management: 1-15.

Agarwal, A. et al., 2016b. Hydrologic regionalization using wavelet-based multiscale entropy method. Journal of Hydrology, 538: 22-32.

AghaKouchak, A., 2014. A baseline probabilistic drought forecasting framework using standardized soil moisture index: application to the 2012 United States drought. Hydrology and Earth System Sciences, 18(7): 2485-2492.

AghaKouchak, A., 2015. A multivariate approach for persistence-based drought prediction: Application to the 2010-2011 East Africa drought. Journal of Hydrology, 526: 127-135.

Ajami, N.K., Duan, Q., Sorooshian, S., 2007. An integrated hydrologic Bayesian multimodel combination framework: Confronting input, parameter, and model structural uncertainty in hydrologic prediction. Water Resources Research, 43(1).

Alapaty, K., Raman, S., Niyogi, D.S., 1997. Uncertainty in the specification of surface characteristics: A study of prediction errors in the boundary layer. Boundary-Layer Meteorology, 82(3): 475-502.

Barnett, T.P. et al., 1999. Origins of the midlatitude Pacific decadal variability. Geophysical Research Letters, 26(10): 1453-1456. 
Beebee, R.A., Manga, M., 2004. Variation in the relationship between snowmelt runoff and ENSO and PDO. Wiley Online Library.

Bell, J.E. et al., 2013. US Climate Reference Network soil moisture and temperature observations. Journal of Hydrometeorology, 14(3): 977-988.

Betts, A.K., Chen, F., Mitchell, K.E., Janjic, Z.I., 1997. Assessment of the land surface and boundary layer models in two operational versions of the NCEP Eta model using FIFE data. Monthly Weather Review, 125(11): 2896-2916.

Box, G.E., Jenkins, G.M., 1976. Time series analysis: forecasting and control, revised ed. Holden-Day.

Brown, M.E. et al., 2013. NASA's soil moisture active passive (SMAP) mission and opportunities for applications users. Bulletin of the American Meteorological Society, 94(8): 1125-1128.

Chen, F., Janjić, Z., Mitchell, K., 1997. Impact of atmospheric surface-layer parameterizations in the new land-surface scheme of the NCEP mesoscale Eta model. Boundary-Layer Meteorology, 85(3): 391-421.

Chen, M., Zhuang, Q., He, Y., 2014. An efficient method of estimating downward solar radiation based on the MODIS observations for the use of land surface modeling. Remote Sensing, 6(8): 7136-7157.

Chou, C.-M., Wang, R.-Y., 2002. On-line estimation of unit hydrographs using the waveletbased LMS algorithm. Hydrological sciences journal, 47(5): 721-738.

Coulibaly, P., Burn, D.H., 2004. Wavelet analysis of variability in annual Canadian streamflows. Water Resources Research, 40(3).

D'Odorico, P., Ridolfi, L., Porporato, A., Rodriguez- Iturbe, I., 2000. Preferential states of seasonal soil moisture: The impact of climate fluctuations. Water Resources Research, 36(8): 2209-2219. 
Dai, A., 2011. Drought under global warming: a review. Wiley Interdisciplinary Reviews: Climate Change, 2(1): 45-65.

Dai, X., Wang, P., Chou, J., 2003. Multiscale characteristics of the rainy season rainfall and interdecadal decaying of summer monsoon in North China. Chinese Science Bulletin, 48(24): 2730-2734.

Daubechies, I., 1990. The wavelet transform, time-frequency localization and signal analysis. IEEE transactions on information theory, 36(5): 961-1005.

Delworth, T.L., Manabe, S., 1988. The influence of potential evaporation on the variabilities of simulated soil wetness and climate. Journal of Climate, 1(5): 523-547.

Dorigo, W. et al., 2013. Global automated quality control of in situ soil moisture data from the International Soil Moisture Network. Vadose Zone Journal, 12(3).

Duan, Q., Ajami, N.K., Gao, X., Sorooshian, S., 2007. Multi-model ensemble hydrologic prediction using Bayesian model averaging. Advances in Water Resources, 30(5): 13711386.

Ek, M. et al., 2003. Implementation of Noah land surface model advances in the National Centers for Environmental Prediction operational mesoscale Eta model. Journal of Geophysical Research: Atmospheres, 108(D22).

Fernandez, C., Ley, E., Steel, M.F., 2001a. Benchmark priors for Bayesian model averaging. Journal of Econometrics, 100(2): 381-427.

Fernandez, C., Ley, E., Steel, M.F., 2001b. Model uncertainty in cross- country growth regressions. Journal of applied Econometrics, 16(5): 563-576.

Ford, T.W., McRoberts, D.B., Quiring, S.M., Hall, R.E., 2015. On the utility of in situ soil moisture observations for flash drought early warning in Oklahoma, USA. Geophysical Research Letters, 42(22): 9790-9798. 
Fragoso, T.M., Neto, F.L., 2015. Bayesian model averaging: A systematic review and conceptual classification. arXiv preprint arXiv:1509.08864.

Fredj, E., Roarty, H., Kohut, J., Smith, M., Glenn, S., 2016. Gap Filling of the Coastal Ocean Surface Currents from HFR Data: Application to the Mid-Atlantic Bight HFR Network. Journal of Atmospheric and Oceanic Technology, 33(6): 1097-1111.

Garcia, D., 2010. Robust smoothing of gridded data in one and higher dimensions with missing values. Computational statistics \& data analysis, 54(4): 1167-1178.

Hao, Z., AghaKouchak, A., 2014. A nonparametric multivariate multi-index drought monitoring framework. Journal of Hydrometeorology, 15(1): 89-101.

Hayes, M., Svoboda, M., Wall, N., Widhalm, M., 2011. The Lincoln declaration on drought indices: universal meteorological drought index recommended. Bulletin of the American Meteorological Society, 92(4): 485-488.

Hoeting, J.A., Madigan, D., Raftery, A.E., Volinsky, C.T., 1999. Bayesian model averaging: a tutorial. Statistical science: $382-401$.

Hunt, E.D., Hubbard, K.G., Wilhite, D.A., Arkebauer, T.J., Dutcher, A.L., 2009. The development and evaluation of a soil moisture index. International Journal of Climatology, 29(5): 747.

Iglesias, A., Garrote, L., Cancelliere, A., Cubillo, F., Wilhite, D.A., 2009. Coping with drought risk in agriculture and water supply systems: Drought management and policy development in the Mediterranean, 26. Springer Science \& Business Media.

Karl, T., Koss, W.J., 1984. Regional and national monthly, seasonal, and annual temperature weighted by area, 1895-1983. National Climatic Data Center.

Karl, T.R., 1983. Some spatial characteristics of drought duration in the United States. Journal of Climate and Applied Meteorology, 22(8): 1356-1366. 
Kim, J., Mohanty, B.P., Shin, Y., 2015. Effective soil moisture estimate and its uncertainty using multimodel simulation based on Bayesian Model Averaging. Journal of Geophysical Research: Atmospheres, 120(16): 8023-8042.

Kim, S., 2004. Wavelet analysis of precipitation variability in northern California, USA. KSCE Journal of Civil Engineering, 8(4): 471-477.

Kim, T.-W., Valdés, J.B., 2003. Nonlinear model for drought forecasting based on a conjunction of wavelet transforms and neural networks. Journal of Hydrologic Engineering, 8(6): 319-328.

Koren, V. et al., 1999. A parameterization of snowpack and frozen ground intended for NCEP weather and climate models. Journal of Geophysical Research: Atmospheres, 104(D16): 19569-19585.

Koster, R.D. et al., 2004. Regions of strong coupling between soil moisture and precipitation. Science, 305(5687): 1138-1140.

Koster, R.D., Suarez, M.J., 1994. The components of a 'SVAT'scheme and their effects on a GCM's hydrological cycle. Advances in Water Resources, 17(1-2): 61-78.

Koster, R.D., Suarez, M.J., 1996. Energy and water balance calculations in the Mosaic LSM.

Kundzewicz, Z.W., Hirabayashi, Y., Kanae, S., 2010. River floods in the changing climateobservations and projections. Water Resources Management, 24(11): 2633-2646.

Lakshmi, V., Piechota, T., Narayan, U., Tang, C., 2004. Soil moisture as an indicator of weather extremes. Geophysical research letters, 31(11).

Leamer, E.E., 1978. Specification searches: Ad hoc inference with nonexperimental data, 53. John Wiley \& Sons Incorporated.

Lu, R., 2002. Decomposition of interdecadal and interannual components for North China rainfall in rainy season. Chinese Journal of Atmosphere (in Chinese), 26: 611-624. 
Luo, L., Wood, E.F., 2007. Monitoring and predicting the 2007 US drought. Geophysical Research Letters, 34(22).

Madadgar, S., Moradkhani, H., 2013. A Bayesian framework for probabilistic seasonal drought forecasting. Journal of Hydrometeorology, 14(6): 1685-1705.

Maheswaran, R., Khosa, R., 2012a. Comparative study of different wavelets for hydrologic forecasting. Computers \& Geosciences, 46: 284-295.

Maheswaran, R., Khosa, R., 2012b. Wavelet-Volterra coupled model for monthly stream flow forecasting. Journal of Hydrology, 450: 320-335.

Mallat, S., 1999. A Wavelet Tour of Signal Processing, (Wavelet Analysis \& Its Applications).

Mishra, A.K., Singh, V.P., 2010. A review of drought concepts. Journal of Hydrology, 391(1): 202-216.

Mitchell, K.E. et al., 2004a. The multi- institution North American Land Data Assimilation System (NLDAS): Utilizing multiple GCIP products and partners in a continental distributed hydrological modeling system. Journal of Geophysical Research: Atmospheres, 109(D7).

Mitchell, K.E. et al., 2004b. The multi- institution North American Land Data Assimilation System (NLDAS): Utilizing multiple GCIP products and partners in a continental distributed hydrological modeling system. Journal of Geophysical Research: Atmospheres (1984-2012), 109(D7).

Mo, K.C., 2008. Model-based drought indices over the United States. Journal of Hydrometeorology, 9(6): 1212-1230.

Mo, K.C., Chen, L.-C., Shukla, S., Bohn, T.J., Lettenmaier, D.P., 2012. Uncertainties in North American land data assimilation systems over the contiguous United States. Journal of Hydrometeorology, 13(3): 996-1009. 
Mozny, M. et al., 2012. Use of a soil moisture network for drought monitoring in the Czech Republic. Theoretical and Applied Climatology, 107(1-2): 99-111.

Nanda, T., Sahoo, B., Beria, H., Chatterjee, C., 2016. A wavelet-based non-linear autoregressive with exogenous inputs (WNARX) dynamic neural network model for realtime flood forecasting using satellite-based rainfall products. Journal of Hydrology, 539: $57-73$.

Narayan, U., Lakshmi, V., 2008. Characterizing subpixel variability of low resolution radiometer derived soil moisture using high resolution radar data. Water resources research, 44(6).

Oglesby, R.J., Marshall, S., Erickson, D.J., Roads, J.O., Robertson, F.R., 2002. Thresholds in atmosphere-soil moisture interactions: Results from climate model studies. Journal of Geophysical Research: Atmospheres, 107(D14).

Otkin, J.A. et al., 2016. Assessing the evolution of soil moisture and vegetation conditions during the 2012 United States flash drought. Agricultural and Forest Meteorology, 218: 230-242.

Parrish, M.A., Moradkhani, H., DeChant, C.M., 2012. Toward reduction of model uncertainty: Integration of Bayesian model averaging and data assimilation. Water Resources Research, 48(3).

Partal, T., Kişi, Ö., 2007. Wavelet and neuro-fuzzy conjunction model for precipitation forecasting. Journal of Hydrology, 342(1): 199-212.

Peterson, T.C., Stott, P.A., Herring, S., 2012. Explaining extreme events of 2011 from a climate perspective. Bulletin of the American Meteorological Society, 93(7): 1041-1067.

Pulwarty, R.S., Sivakumar, M.V., 2014. Information systems in a changing climate: Early warnings and drought risk management. Weather and Climate Extremes, 3: 14-21.

Quiring, S.M. et al., 2015. The North American soil moisture database: development and applications. Bulletin of the American Meteorological Society(2015). 
Raftery, A.E., Gneiting, T., Balabdaoui, F., Polakowski, M., 2005. Using Bayesian model averaging to calibrate forecast ensembles. Monthly Weather Review, 133(5): 1155-1174.

Rathinasamy, M., Adamowski, J., Khosa, R., 2013. Multiscale streamflow forecasting using a new Bayesian Model Average based ensemble multi-wavelet Volterra nonlinear method. Journal of Hydrology, 507: 186-200.

Robinson, J., Hubbard, K., 1990. Soil water assessment model for several crops in the High Plains. Agronomy Journal, 82(6): 1141-1148.

Rojas, R., Feyen, L., Dassargues, A., 2008. Conceptual model uncertainty in groundwater modeling: Combining generalized likelihood uncertainty estimation and Bayesian model averaging. Water Resources Research, 44(12).

Sahay, R.R., Sehgal, V., 2014. Wavelet-ANFIS models for forecasting monsoon flows: Case study for the Gandak River (India). Water Resources, 41(5): 574-582.

Sehgal, Tiwari, M.K., Chandranath, C., 2014a. Wavelet bootstrap multiple linear regression based hybrid modeling for daily river discharge forecasting. Water resources management, 28(10): 2793-2811.

Sehgal, V., Lakhanpal, A., Maheswaran, R., Khosa, R., Sridhar, V., 2016. Application of multi scale wavelet entropy and multi-resolution Volterra models for climatic downscaling: a case study of Krishna basin, India. Journal of Hydrology, Under Review.

Sehgal, V., Sahay, R.R., Chatterjee, C., 2014b. Effect of utilization of discrete wavelet components on flood forecasting performance of wavelet based ANFIS models. Water resources management, 28(6): 1733-1749.

Sehgal, V., Tiwari, M.K., Chatterjee, C., 2014c. Wavelet bootstrap multiple linear regression based hybrid modeling for daily river discharge forecasting. Water resources management, 28(10): 2793-2811. 
Sheffield, J., Goteti, G., Wen, F., Wood, E.F., 2004a. A simulated soil moisture based drought analysis for the United States. Journal of Geophysical Research: Atmospheres (1984-2012), 109(D24).

Sheffield, J., Goteti, G., Wen, F., Wood, E.F., 2004b. A simulated soil moisture based drought analysis for the United States. Journal of Geophysical Research: Atmospheres, 109(D24).

Sheffield, J. et al., 2014. A drought monitoring and forecasting system for sub-Sahara African water resources and food security. Bulletin of the American Meteorological Society, 95(6): 861-882.

Sims, A.P., Raman, S., 2002. Adopting drought indices for estimating soil moisture: A North Carolina case study. Geophysical Research Letters, 29(8).

Smith, L.C., Turcotte, D.L., Isacks, B.L., 1998. Stream flow characterization and feature detection using a discrete wavelet transform. Hydrological processes, 12(2): 233-249.

Sohrabi, M.M., Ryu, J.H., Abatzoglou, J., Tracy, J., 2015. Development of soil moisture drought index to characterize droughts. Journal of Hydrologic Engineering, 20(11): 04015025 .

Sridhar, V., Hubbard, K., 2009. Estimation of the water balance using observed soil water in the Nebraska sandhills. Journal of Hydrologic Engineering, 15(1): 70-78.

Sridhar, V., Hubbard, K.G., You, J., Hunt, E.D., 2008. Development of the soil moisture index to quantify agricultural drought and its "user friendliness" in severity-area-duration assessment. Journal of Hydrometeorology, 9(4): 660-676.

Sridhar, V. et al., 2013. Evaluating bias-corrected AMSR-E soil moisture using in situ observations and model estimates. Vadose Zone Journal, 12(3).

Sridhar, V., Wedin, D.A., 2009. Hydrological behaviour of grasslands of the Sandhills of Nebraska: water and energy- balance assessment from measurements, treatments, and modelling. Ecohydrology, 2(2): 195-212. 
Su, B., Wang, A., Wang, G., Wang, Y., Jiang, T., 2016. Spatiotemporal variations of soil moisture in the Tarim River basin, China. International Journal of Applied Earth Observation and Geoinformation, 48: 122-130.

Svoboda, M., LeComte, D., Hayes, M., Heim, R., 2002. The drought monitor. Bulletin of the American Meteorological Society, 83(8): 1181.

Tang, C., Piechota, T.C., 2009. Spatial and temporal soil moisture and drought variability in the Upper Colorado River Basin. Journal of Hydrology, 379(1): 122-135.

Torrence, C., Webster, P.J., 1998. The annual cycle of persistence in the El Nño/Southern Oscillation. Quarterly Journal of the Royal Meteorological Society, 124(550): 1985-2004.

Torrence, C., Webster, P.J., 1999. Interdecadal changes in the ENSO-monsoon system. Journal of Climate, 12(8): 2679-2690.

Trenberth, K., Overpeck, J., Solomon, S., 2004. Exploring drought and its implications for the future. Eos, Transactions American Geophysical Union, 85(3): 27-27.

Trenberth, K.E., 1984. Some effects of finite sample size and persistence on meteorological statistics. Part I: Autocorrelations. Monthly Weather Review, 112(12): 2359-2368.

Trenberth, K.E., Fasullo, J.T., 2012. Climate extremes and climate change: The Russian heat wave and other climate extremes of 2010. Journal of Geophysical Research: Atmospheres, 117(D17).

Vrugt, J.A., Robinson, B.A., 2007. Treatment of uncertainty using ensemble methods: Comparison of sequential data assimilation and Bayesian model averaging. Water Resources Research, 43(1).

Wang, A., Bohn, T.J., Mahanama, S.P., Koster, R.D., Lettenmaier, D.P., 2009. Multimodel ensemble reconstruction of drought over the continental United States. Journal of Climate, 22(10): 2694-2712. 
Wang, A., Lettenmaier, D.P., Sheffield, J., 2011. Soil moisture drought in China, 1950-2006. Journal of Climate, 24(13): 3257-3271.

Wang, A., Zeng, X., Shen, S.S., Zeng, Q.-C., Dickinson, R.E., 2006. Time scales of land surface hydrology. Journal of Hydrometeorology, 7(5): 868-879.

Wang, G., Garcia, D., Liu, Y., De Jeu, R., Dolman, A.J., 2012. A three-dimensional gap filling method for large geophysical datasets: Application to global satellite soil moisture observations. Environmental Modelling \& Software, 30: 139-142.

Wang, W., Ding, J., 2003. Wavelet network model and its application to the prediction of hydrology. Nature and Science, 1(1): 67-71.

Wilhite, D.A., Glantz, M.H., 1985. Understanding: the drought phenomenon: the role of definitions. Water international, 10(3): 111-120.

Wood, A.W., 2008. The University of Washington Surface Water Monitor: An experimental platform for national hydrologic assessment and prediction, American Meteorology Society annual meeting, 22nd conference on hydrology, New Orleans. p.

Wood, A.W., Maurer, E.P., Kumar, A., Lettenmaier, D.P., 2002. Long- range experimental hydrologic forecasting for the eastern United States. Journal of Geophysical Research: Atmospheres, 107(D20).

Xia, Y. et al., 2014. Uncertainties, correlations, and optimal blends of drought indices from the NLDAS multiple land surface model ensemble. Journal of Hydrometeorology, 15(4): $1636-1650$.

Xia, Y. et al., 2012. Continental- scale water and energy flux analysis and validation for the North American Land Data Assimilation System project phase 2 (NLDAS- 2): 1. Intercomparison and application of model products. Journal of Geophysical Research: Atmospheres, 117(D3).

Yuan, X. et al., 2013. Probabilistic seasonal forecasting of African drought by dynamical models. Journal of Hydrometeorology, 14(6): 1706-1720. 
Zhang, L., Zhang, H., Zhang, Q., Li, Y., Zhao, J., 2016. On the potential application of land surface models for drought monitoring in China. Theoretical and Applied Climatology: 117. DOI:10.1007/s00704-016-1730-0 


\section{Tables}

Table 1: Description of layer thickness for Noah and Mosaic land surface models; and sensor location for in-situ observations at US Climate Reference Network (USCRN) stations ............... 1 Table 2: Model performance of WBR models with the best performing WR models for the total

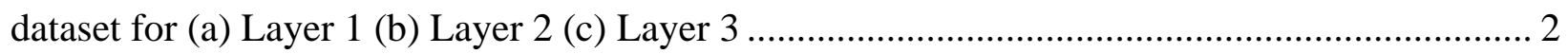
Table 3: Performance evaluation of the proposed models for the training and validation dataset for $\mathrm{WBR}_{\mathrm{Hybrid}}$ and best performing Wavelet Regression (WR) models for each layer................... 3

Table 4: A brief description of the models explored in this study ............................................ 3

Table 5: Performance of models developed for comparison with WBR Hybrid models for total

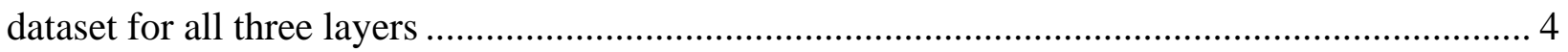

Table 7: U.S. Drought Monitor (USDM) classification of drought based on soil moisture

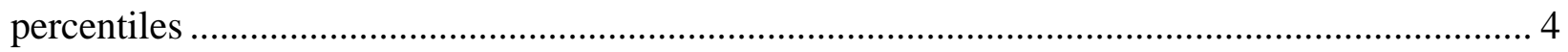

Table 8: Classification of drought based on layer- wise soil moisture percentiles proposed in this study ... 4

Table 1: Description of layer thickness for Noah and Mosaic land surface models; and sensor location for in-situ observations at US Climate Reference Network (USCRN) stations

\begin{tabular}{|c|c|c|c|c|c|c|}
\hline \multirow{2}{*}{ Model/Source } & \multicolumn{6}{|c|}{ Layer / sensor depth description for Noah, Mosaic and USCRN data } \\
\hline & \multicolumn{6}{|c|}{ Soil column thickness available in model (in $\mathrm{cm}$ ) } \\
\hline Noah & $0-10$ & $10-40$ & $0-40$ & $40-200$ & $0-100$ & $0-200$ \\
\hline \multirow{2}{*}{ Mosaic } & $0-10$ & $10-40$ & $40-100$ & $0-100$ & $0-200$ & $100-200$ \\
\hline & \multicolumn{6}{|c|}{ Sensor depth from surface (in cm) } \\
\hline \multirow[t]{2}{*}{ USCRN } & 5 & 10 & 20 & 50 & 100 & \\
\hline & \multicolumn{6}{|c|}{ Layer thickness / interpolated sensor depth used in the study } \\
\hline Noah/ Mosaic & $0-10$ & $10-40$ & $40-100$ & & & \\
\hline Interpolated USCRN & 5 & 25 & 75 & & & \\
\hline
\end{tabular}


Table 2: Model performance of WBR models with the best performing WR models for the total dataset for (a) Layer 1 (b) Layer 2 (c) Layer 3

\begin{tabular}{lllll}
\hline \multicolumn{5}{c}{ (a) Model performance for total data- Layer 1 } \\
\hline Model & CC & E & NRMSE & Weights \\
\hline$W R(7)$ & 0.7937 & 0.6298 & 0.1423 & 0.072 \\
$W R(3)$ & 0.7930 & 0.6286 & 0.1425 & 0.252 \\
$W R(11)$ & 0.7873 & 0.6192 & 0.1443 & 0.024 \\
$W R(24)$ & 0.7862 & 0.6180 & 0.1446 & 0.003 \\
$W R(33)$ & 0.7854 & 0.6168 & 0.1448 & 0.252 \\
$W R(28)$ & 0.7842 & 0.6148 & 0.1451 & 0.048 \\
$W R(15)$ & 0.7842 & 0.6147 & 0.1452 & 0.006 \\
$W R(20)$ & 0.7840 & 0.6145 & 0.1452 & 0.006 \\
$W R(37)$ & 0.7822 & 0.6118 & 0.1457 & 0.087 \\
$W R(16)$ & 0.7802 & 0.6084 & 0.1464 & 0.252 \\
\hline$W B R_{\text {Hybrid }}$ & 0.7924 & 0.6272 & 0.1428 & \\
\hline \multicolumn{5}{c}{}
\end{tabular}

\begin{tabular}{lllll}
\hline \multicolumn{5}{l}{ (b) Model performance for total data- Layer 2 } \\
\hline Model & CC & E & NRMSE & Weights \\
\hline$W E(3)$ & 0.7596 & 0.5765 & 0.1582 & 0.167 \\
$W R(43)$ & 0.7570 & 0.5728 & 0.1589 & 0.167 \\
$W R(7)$ & 0.7549 & 0.5696 & 0.1594 & 0.053 \\
$W R(33)$ & 0.7548 & 0.5695 & 0.1595 & 0.167 \\
$W R(29)$ & 0.7545 & 0.5692 & 0.1595 & 0.060 \\
$W R(38)$ & 0.7545 & 0.5690 & 0.1596 & 0.166 \\
$W R(42)$ & 0.7538 & 0.5680 & 0.1597 & 0.003 \\
$W R(34)$ & 0.7536 & 0.5677 & 0.1598 & 0.004 \\
$W R(25)$ & 0.7522 & 0.5657 & 0.1602 & 0.047 \\
$W R(37)$ & 0.7521 & 0.5654 & 0.1602 & 0.167 \\
\hline$W B R_{\text {Hybrid }}$ & 0.7661 & 0.5855 & 0.1565 & \\
\hline \multicolumn{5}{l}{} \\
\end{tabular}

(c) Model performance for total data-Layer 3

\begin{tabular}{lllll}
\hline Model & CC & E & NRMSE & Weights \\
\hline$W R(42)$ & 0.7500 & 0.5616 & 0.1553 & 0.415 \\
$W R(37)$ & 0.7466 & 0.5567 & 0.1562 & 0.039 \\
$W R(33)$ & 0.7455 & 0.5548 & 0.1565 & 0.041 \\
$W R(29)$ & 0.7454 & 0.5551 & 0.1564 & 0.011 \\
$W R(24)$ & 0.7446 & 0.5537 & 0.1567 & 0.024 \\
$W R(41)$ & 0.7445 & 0.5535 & 0.1567 & 0.161 \\
$W R(38)$ & 0.7444 & 0.5531 & 0.1568 & 0.022 \\
$W R(20)$ & 0.7444 & 0.5536 & 0.1567 & 0.052 \\
$W R(28)$ & 0.7436 & 0.5523 & 0.1569 & 0.167 \\
$W R(16)$ & 0.7427 & 0.5507 & 0.1572 & 0.070 \\
\hline$W B R_{\text {Hybrid }}$ & 0.7505 & 0.5619 & 0.1552 &
\end{tabular}


Table 3: Performance evaluation of the proposed models for the training and validation dataset for $\mathrm{WBR}_{\text {Hybrid }}$ and best performing Wavelet Regression (WR) models for each layer.

\begin{tabular}{|c|c|c|c|c|c|c|}
\hline & \multicolumn{2}{|l|}{ Layer 1} & \multicolumn{2}{|l|}{ Layer 2} & \multicolumn{2}{|l|}{ Layer 3} \\
\hline & \multicolumn{5}{|c|}{ Training } & \multirow[b]{2}{*}{ WR } \\
\hline & WBR $_{\text {Hybrid }}$ & WR & WBR $_{\text {Hybrid }}$ & WR & WBR $_{\text {Hybrid }}$ & \\
\hline Correlation & 0.80 & 0.79 & 0.76 & 0.74 & 0.75 & 0.75 \\
\hline $\mathrm{E}$ & 0.64 & 0.64 & 0.59 & 0.56 & 0.57 & 0.57 \\
\hline \multirow[t]{3}{*}{ NRMSE } & 0.141 & 0.142 & 0.159 & 0.165 & 0.157 & 0.157 \\
\hline & \multicolumn{6}{|c|}{ Validation } \\
\hline & WBR $_{\text {Hybrid }}$ & WR & WBR $_{\text {Hybrid }}$ & WR & WBR $_{\text {Hybrid }}$ & WR \\
\hline Correlation & 0.78 & 0.78 & 0.75 & 0.70 & 0.73 & 0.71 \\
\hline $\mathrm{E}$ & 0.60 & 0.59 & 0.57 & 0.48 & 0.53 & 0.50 \\
\hline NRMSE & 0.150 & 0.152 & 0.159 & 0.174 & 0.154 & 0.160 \\
\hline
\end{tabular}

Table 4: A brief description of the models explored in this study

\begin{tabular}{|c|c|c|c|c|}
\hline Model name & $\begin{array}{l}\text { Input } \\
\text { variables }\end{array}$ & Modelling methodology & $\begin{array}{l}\text { Wavelet } \\
\text { Regression }\end{array}$ & BMA \\
\hline$W B R_{\text {Hybrid }}$ & $\begin{array}{l}\text { Noah }+ \\
\text { Mosaic }\end{array}$ & $\begin{array}{l}\text { Noah and Mosaic inputs fed to WR modelling scheme } \\
\text { followed by BMA }\end{array}$ & Yes & Yes \\
\hline$W B R_{\text {Noah }}$ & Noah & $\begin{array}{l}\text { Noah inputs fed to WR modelling scheme followed by } \\
\text { BMA }\end{array}$ & Yes & Yes \\
\hline$W B R_{\text {Mosaic }}$ & Mosaic & $\begin{array}{l}\text { Mosaic inputs fed to WR modelling scheme followed } \\
\text { by BMA }\end{array}$ & Yes & Yes \\
\hline$W B R_{\text {Average }}$ & $\begin{array}{l}\text { Noah }+ \\
\text { Mosaic }\end{array}$ & $\begin{array}{l}\text { Noah and Mosaic fed to two separate WR+ BMA } \\
\text { schemes and the outputs are averaged }\end{array}$ & Yes & Yes \\
\hline Noah & Noah & Original Noah dataset & No & No \\
\hline Mosaic & Mosaic & Original Mosaic dataset & No & No \\
\hline
\end{tabular}


Table 5: Performance of models developed for comparison with $\mathrm{WBR}_{\text {Hybrid }}$ models for total dataset for all three layers

\begin{tabular}{llllll}
\cline { 2 - 6 } & \multicolumn{5}{c}{ Model performance for total data } \\
\cline { 2 - 6 } & Noah & Mosaic & WBR $_{\text {Noah }}$ & WBR $_{\text {Mosaic }}$ & WBR $_{\text {Average }}$ \\
\cline { 2 - 6 } Correlation & 0.705 & 0.685 & 0.740 & 0.739 & 0.770 \\
E & 0.361 & 0.402 & 0.547 & 0.546 & 0.588 \\
NRMSE & 0.187 & 0.181 & 0.157 & 0.158 & 0.150 \\
\hline \multirow{5}{*}{ Noah } & Mosaic & WBR & Nayer 2 \\
& 0.665 & 0.681 & 0.708 & 0.726 & 0.744 \\
\hline Correlation & 0.405 & 0.430 & 0.502 & 0.528 & 0.550 \\
E & 0.181 & 0.177 & 0.165 & 0.161 & 0.157 \\
NRMSE & \multicolumn{5}{c}{ Layer 3} \\
& Noah & Mosaic & WBR & Woah \\
& 0.559 & 0.665 & 0.626 & 0.696 & 0.699 \\
\hline Correlation & 0.272 & 0.439 & 0.391 & 0.485 & 0.481 \\
E & 0.222 & 0.195 & 0.203 & 0.186 & 0.187 \\
NRMSE & \multicolumn{5}{c}{ Wosaic } \\
\hline
\end{tabular}

Table 6: U.S. Drought Monitor (USDM) classification of drought based on soil moisture percentiles

\begin{tabular}{lll}
\hline Soil Moisture Percentile & USDM classification & Interpretation \\
\hline Less than 30 & D0 & Abnormally dry \\
Less than 20 & D1 & Moderate drought \\
Less than 10 & D2 & Severe drought \\
Less than 5 & D3 & Extreme drought \\
Less than 2 & D4 & Exceptional drought \\
\hline
\end{tabular}

Table 7: Classification of drought based on layer- wise soil moisture percentiles proposed in this study

\begin{tabular}{llll}
\hline \multicolumn{4}{c}{ Proposed layer- wise classification of drought severity } \\
\hline Soil Moisture Percentile & Layer 1 & Layer 2 & Layer 3 \\
\hline Less than 30 & D0 & D0 & D2 \\
Less than 20 & D0 & D1 & D2 \\
Less than 10 & D0 & D1 & D2 \\
Less than 5 & D0 & D2 & D3 \\
Less than 2 & D0 & D3 & D4 \\
\hline \multicolumn{4}{c}{$*$ Interpretation of $D 0, D 1, D 2, D 3, D 4$ is consistent with USDM }
\end{tabular}




\section{List of Figures}

Fig. 1: (a) Location of the U.S. Climate reference network (USCRN) stations across US. The red triangle indicates no data is currently available for those stations. Blue circle indicates stations with data available for all five layers. Grey circle indicates control stations. (b) Nine climate regions of the U.S. $(C=$ Central, UMW=Upper Midwest, $\mathrm{NE}=$ North East, $\mathrm{NW}=$ North West, $\mathrm{S}=\mathrm{South}, \mathrm{SE}=$ South East, $\mathrm{SW}=$ South West, W= West, WNC= West North Central).

Fig. 2: Observed in-situ soil moisture data (in red) with interpolated gaps (in blue) at daily time step for five sensor depths. Each plot represents the station with maximum number of missing values (less than the permissible limit of $15 \%$ ) for a given sensor depth 5

Fig. 3: Flowchart for the wavelet-based multi- resolution regression (WR) models. Forty-five WR models are developed corresponding to each $d b$ wavelet with vanishing moments from 1 to 45 individually for each layer.

Fig. 4: Wavelet spectrums of the observed in- situ soil moisture time series from the control stations for training dataset using Morlet wavelet. The thick black contour delineates the 5\% significance level against the red noise. Red and blue colours represent stronger and weaker power respectively. It can be observed that the period around 256- 512 is significant for all three layers.

Fig. 5: Flowchart for the Bayesian Model Averaging (BMA) of top performing wavelet-based multiresolution regression (WR) models to obtain Hybrid Soil Moisture. This process is carried out individually for each layer.

Fig. 6: Percentage of in- situ observations being over (O)/ satisfactorily(S) / under (U) estimated for each control station by Noah and Mosaic LSMs.

Fig. 7: Scatter plots with respective Root Mean Square Error (RMSE) of observed soil moisture v/s Hybrid Soil Moisture, Noah and Mosaic models for all three layers for (a) Validation dataset (b) Total dataset 9

Fig. 8: Histograms for correlation of the observed in- situ soil moisture from control stations with HSM, Noah and Mosaic for the three layers .10 
Fig. 9: Wavelet spectrums of (a) Observed in- situ soil moisture (b) Hybrid Soil Moisture (c) Noah (d) Mosaic time series from the control stations for total dataset for layer 1 using Morlet wavelet. The thick black contour delineates the 5\% significance level against the red noise. Red and blue colours represent stronger and weaker power respectively

Fig. 10: Comparison of op soil layer estimation from SMAP, HSM, Noah and Mosaic for June and July 2015.

Fig. 11: Spatial distribution of Response Time $\left(T_{o}\right)$ of Hybrid Soil Moisture (in months) for the three layers. Red/ Orange represents low persistence whereas Blue/ Cyan represents regions with higher Persistence. .13

Fig. 12: Mean Response time $\left(T_{o}\right)$ for three soil layers for the nine climate regions of the US $(\mathrm{C}=$ Central, UMW=Upper Midwest, NE= North East, NW= North West, $\mathrm{S}=$ South, SE= South East, $\mathrm{SW}=$ South West, W= West, WNC= West North Central) 14

Fig. 13: Correlation plots of Hybrid Soil Moisture with the eight drought indices for the three soil layers for CONUS. Red color represents stronger positive correlation whereas Blue color represents near low or negative correlation with the drought indices. 15

Fig. 14: Mean correlation of soil moisture with drought indices (DIs) for the nine climate regions of the U.S. $(\mathrm{C}=$ Central, UMW=Upper Midwest, $\mathrm{NE}=$ North East, $\mathrm{NW}=$ North West, $\mathrm{S}=\mathrm{South}, \mathrm{SE}=$ South East, $\mathrm{SW}=$ South West, W= West, WNC= West North Central). .16

Fig. 15: Correlation plots of pre- whitened Hybrid Soil Moisture with pre- whitened Z- index (top row) and SPI-1 (bottom row) for the three soil layers for CONUS. Red color represents stronger positive correlation whereas Blue color represents near low or negative correlation with the drought indices. .16

Fig. 16: The Averaged Squared Correlation normalized across three soil layers (Read top to bottom) to obtain the Normalized Averaged Squared Correlation (NASC) for both in- situ US Climate reference network (USCRN) data and Hybrid Soil Moisture for the control stations. NASC $=1$ indicates strongest correlation, $\mathrm{NASC}=0$ indicates weakest, and, $0<\mathrm{NASC}<1$ indicates intermediate correlation between the soil moisture of the respective layer and a given drought index. 
Fig. 17: Reconstructed drought maps using the proposed layer-wise soil moisture percentile approach using HSM v/s USDM drought maps for CONUS (Apr'-11 to Dec'- 11) 18

Fig. 18: Reconstructed drought maps using the proposed layer-wise soil moisture percentile approach using Noah and Mosaic dataset v/s USDM drought maps for CONUS (Apr'-11 to Dec'- 11)...... 19 Fig. 19: Comparison of the area under various drought severities as obtained from the reconstructed drought maps using (a) HSM (b) Noah (c) Mosaic and (d) USDM maps (Apr'-11 to Dec'- 11) for Southern U.S. .20

Fig. 20: Comparison of average value of the eight drought indices (Left) (a) Z- index, SPI-1 and SPI6 months (b) SPI- 9 and SPI- 12 (c) SPI- 24, PDSI and PHDI with monthly soil moisture percentiles obtained using HSM, Noah and Mosaic for (d) Layer 1 (b) Layer2 and (c) Layer 3 for Southern US. 
(a)
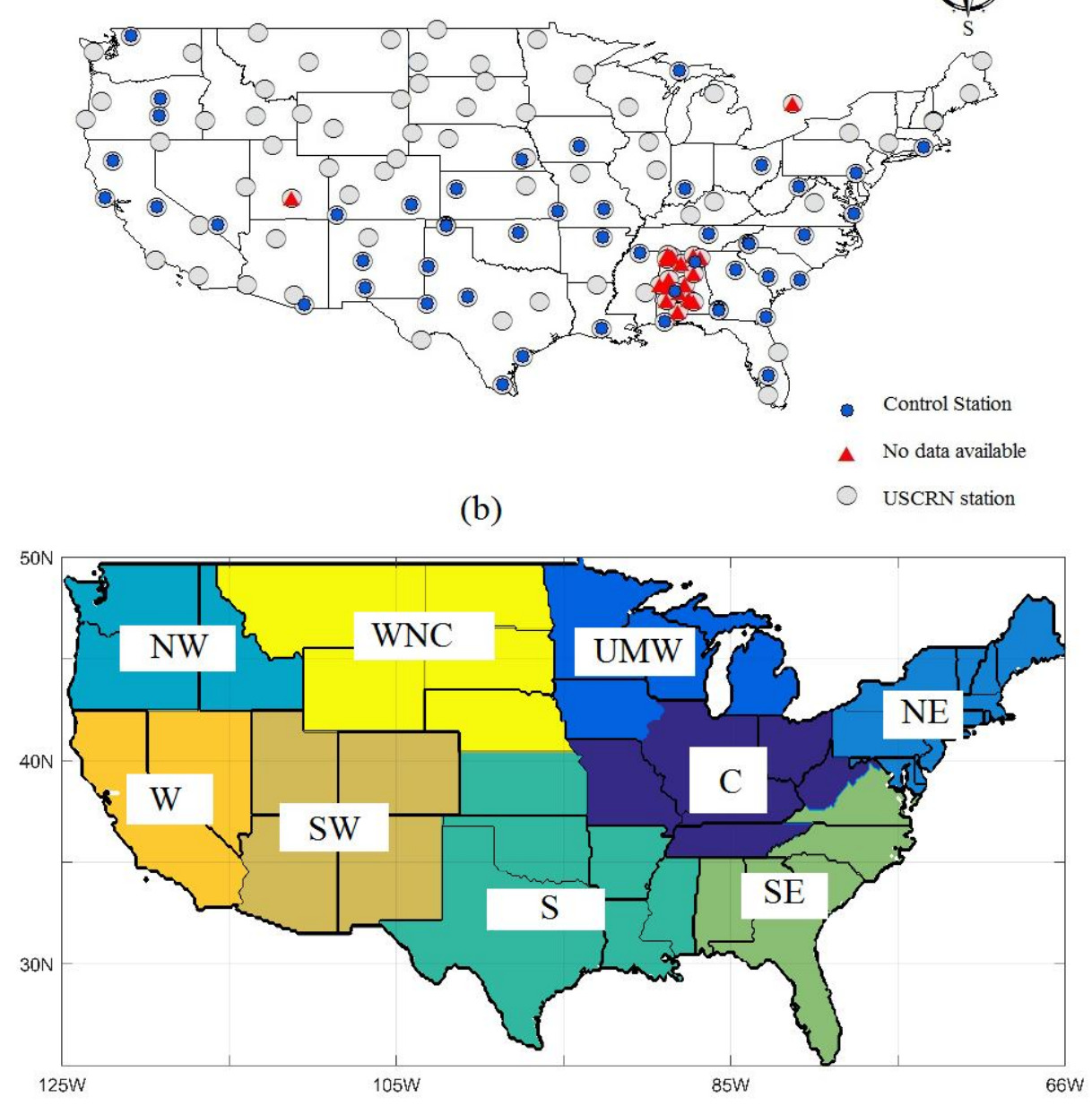

Fig. 1: (a) Location of the U.S. Climate reference network (USCRN) stations across US. The red triangle indicates no data is currently available for those stations. Blue circle indicates stations with data available for all five layers. Grey circle indicates control stations. (b) Nine climate regions of the U.S. $(\mathrm{C}=$ Central, $\mathrm{UMW}=\mathrm{Upper}$ Midwest, $\mathrm{NE}=$ North East, $\mathrm{NW}=$ North West, $\mathrm{S}=$ South, $\mathrm{SE}=$ South East, $\mathrm{SW}=$ South West, W= West, WNC $=$ West North Central). 

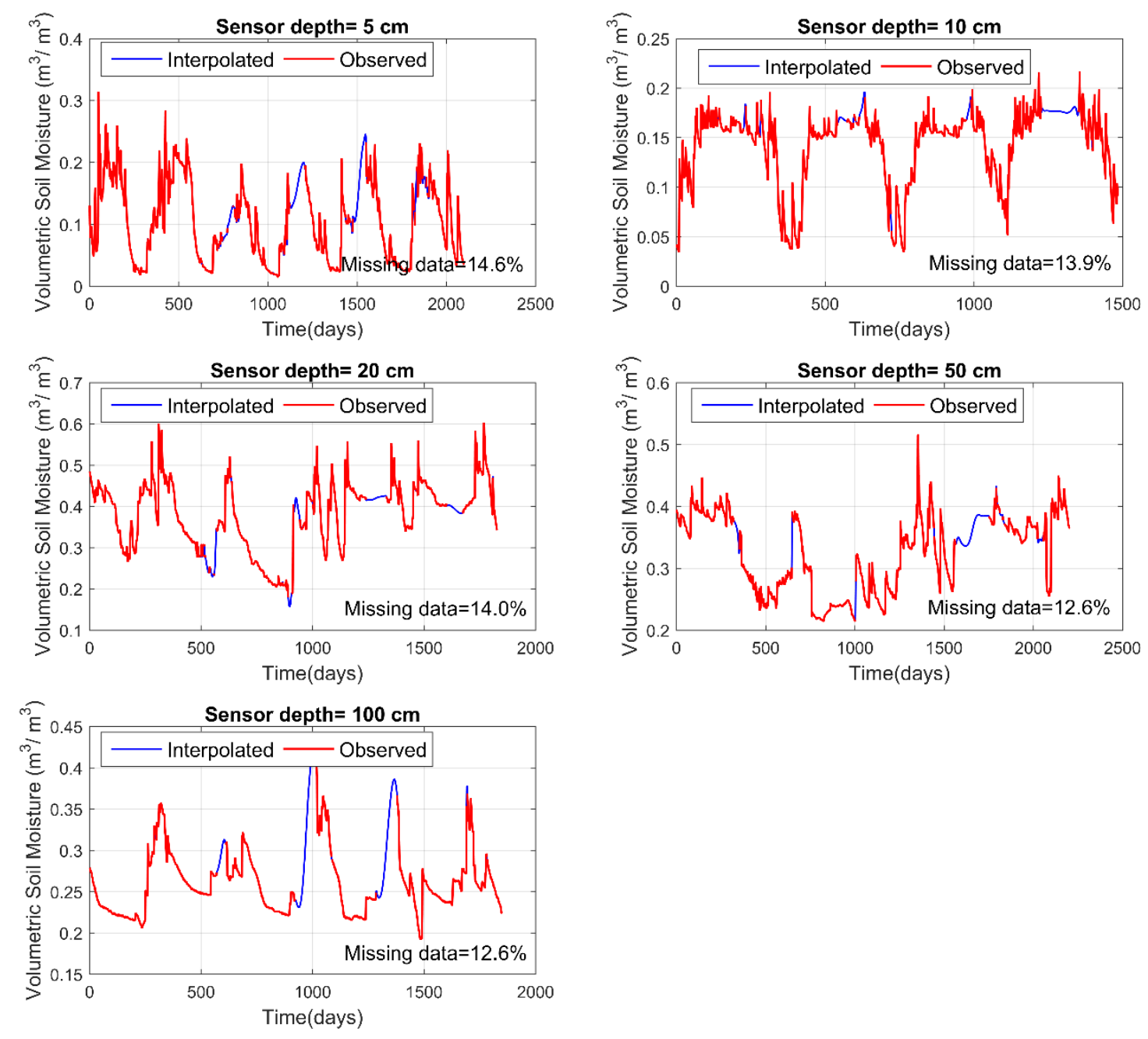

Fig. 2: Observed in- situ soil moisture data (in red) with interpolated gaps (in blue) at daily time step for five sensor depths. Each plot represents the station with maximum number of missing values (less than the permissible limit of 15\%) for a given sensor depth. 


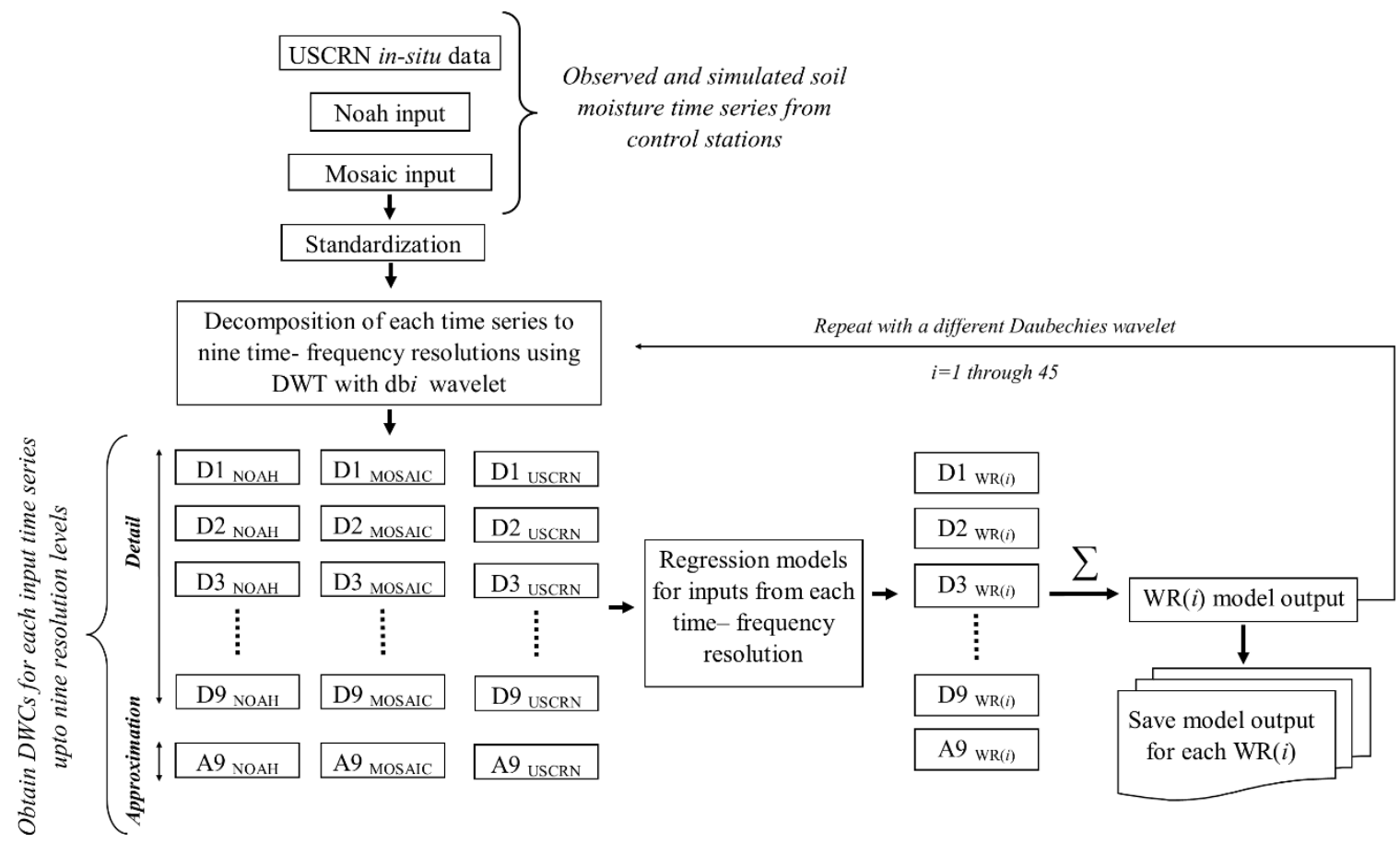

Fig. 3: Flowchart for the wavelet-based multi- resolution regression (WR) models. Forty-five WR models are developed corresponding to each $d b$ wavelet with vanishing moments from 1 to 45 individually for each layer.
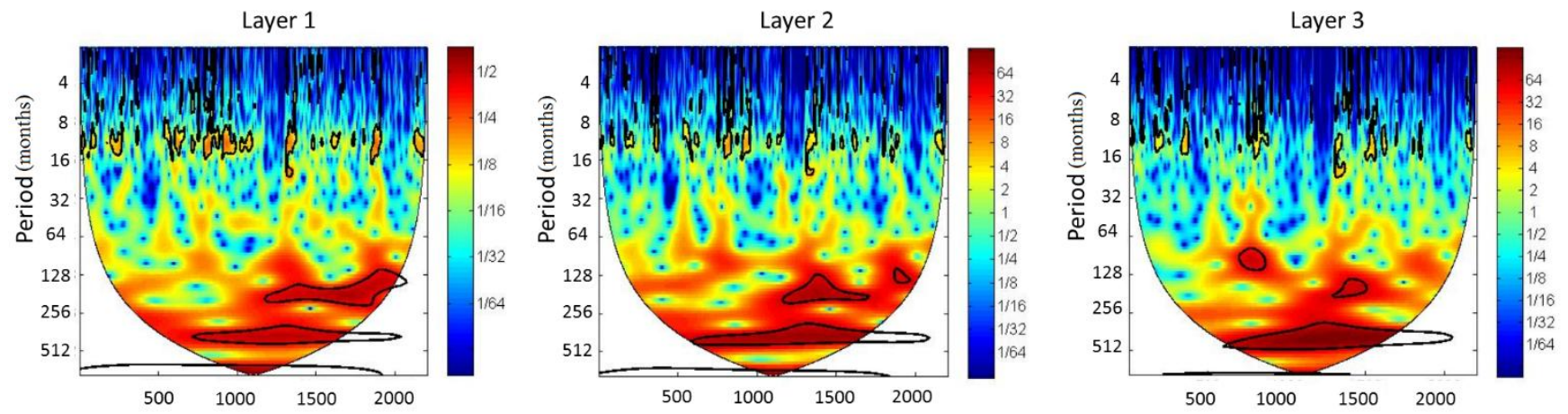

Fig. 4: Wavelet spectrums of the observed in- situ soil moisture time series from the control stations for training dataset using Morlet wavelet. The thick black contour delineates the 5\% significance level against the red noise. Red and blue colours represent stronger and weaker power respectively. It can be observed that the period around 256- 512 is significant for all three layers. 
Outputs of WR models using different Daubechies wavelets for decomposition of input variables

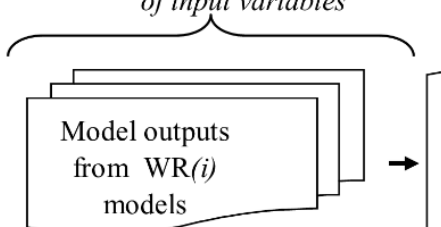

Where $i=1$ through 45

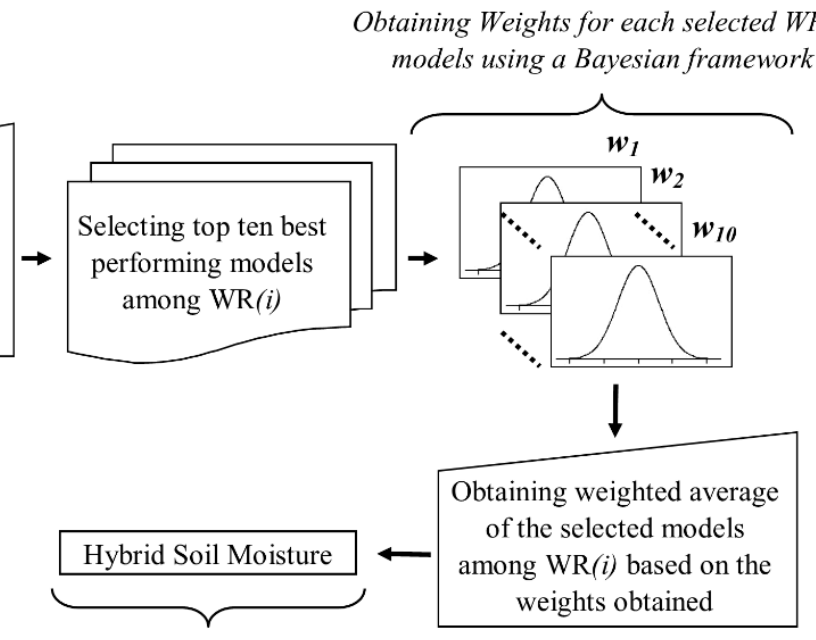

WBR ${ }_{\text {Hybrid }}$ output
Ranking of WR $(i)$ models based on correlation with observed dataset

Fig. 5: Flowchart for the Bayesian Model Averaging (BMA) of top performing wavelet-based multi- resolution regression (WR) models to obtain Hybrid Soil Moisture. This process is carried out individually for each layer. 

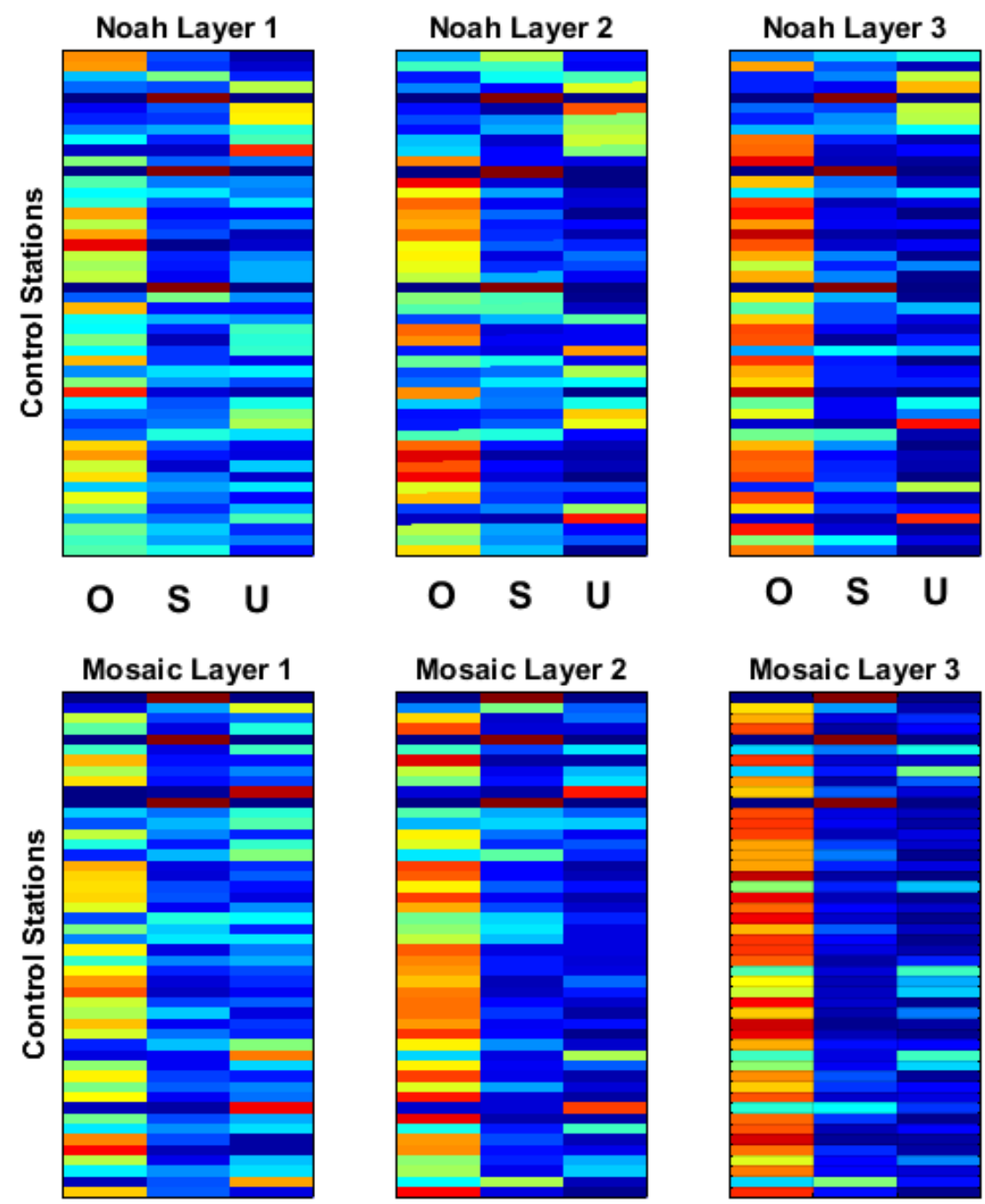

O S U

O S U

O S U

$\%$ of total observations for each control station

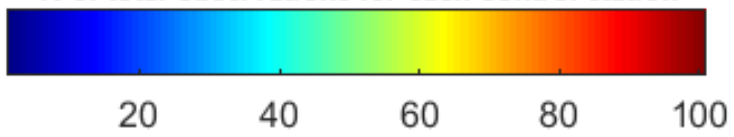

Fig. 6: Percentage of in- situ observations being over (O)/ satisfactorily(S) / under (U) estimated for each control station by Noah and Mosaic LSMs. 

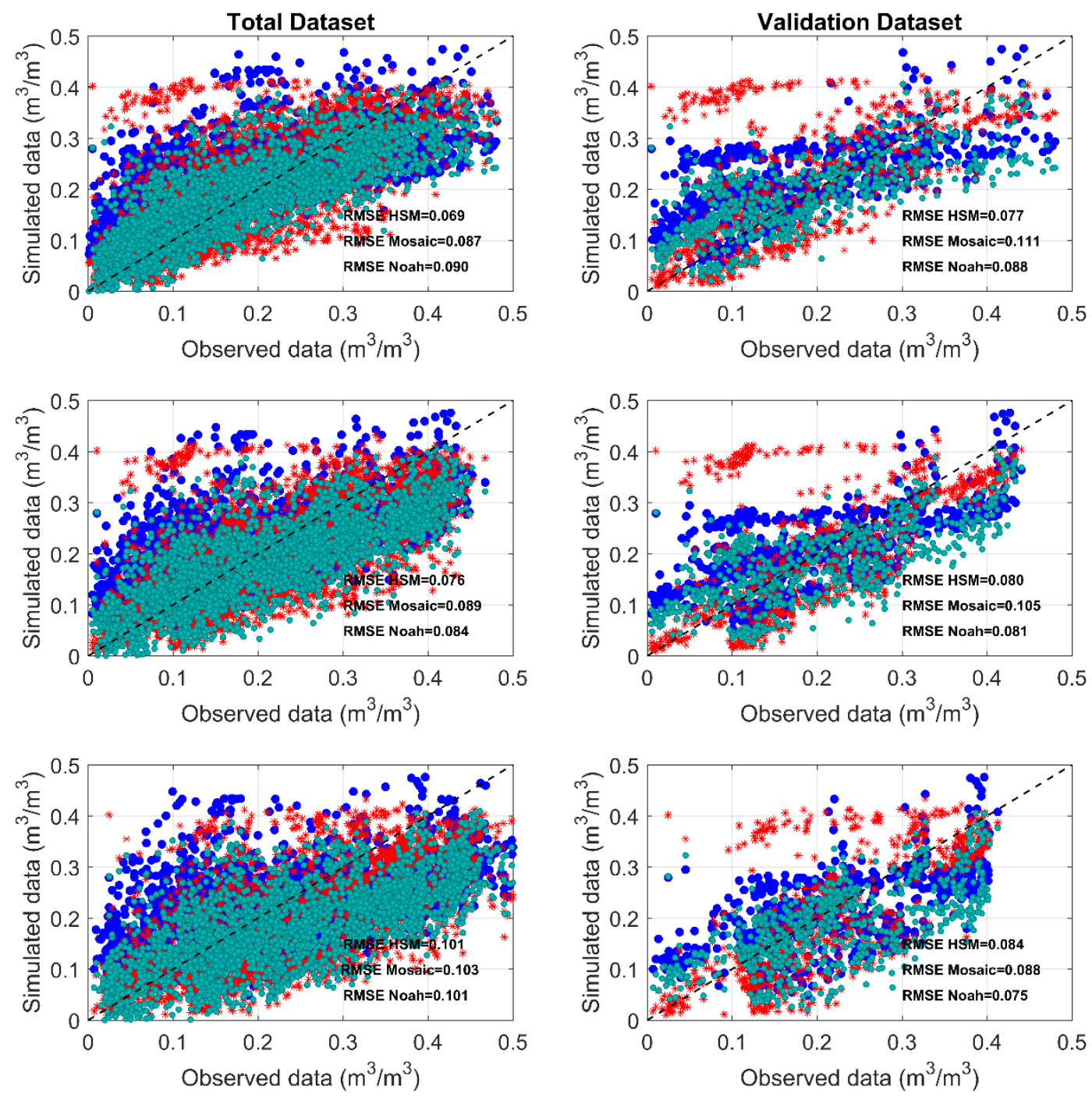

- Noah * Mosaic • HSM

Fig. 7: Scatter plots with respective Root Mean Square Error (RMSE) of observed soil moisture v/s Hybrid Soil Moisture, Noah and Mosaic models for all three layers for (a)

Validation dataset (b) Total dataset 

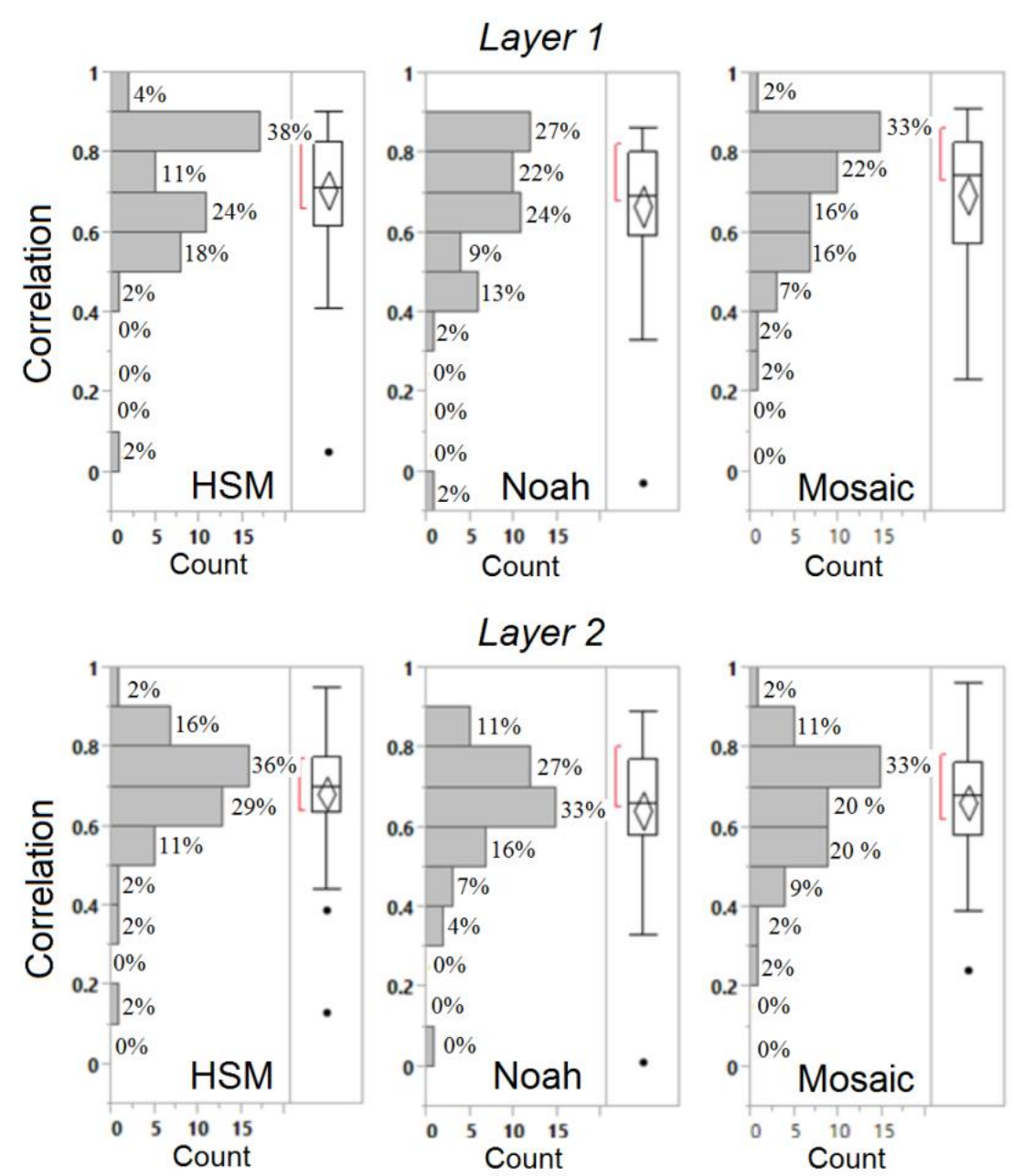

\section{Layer 3}
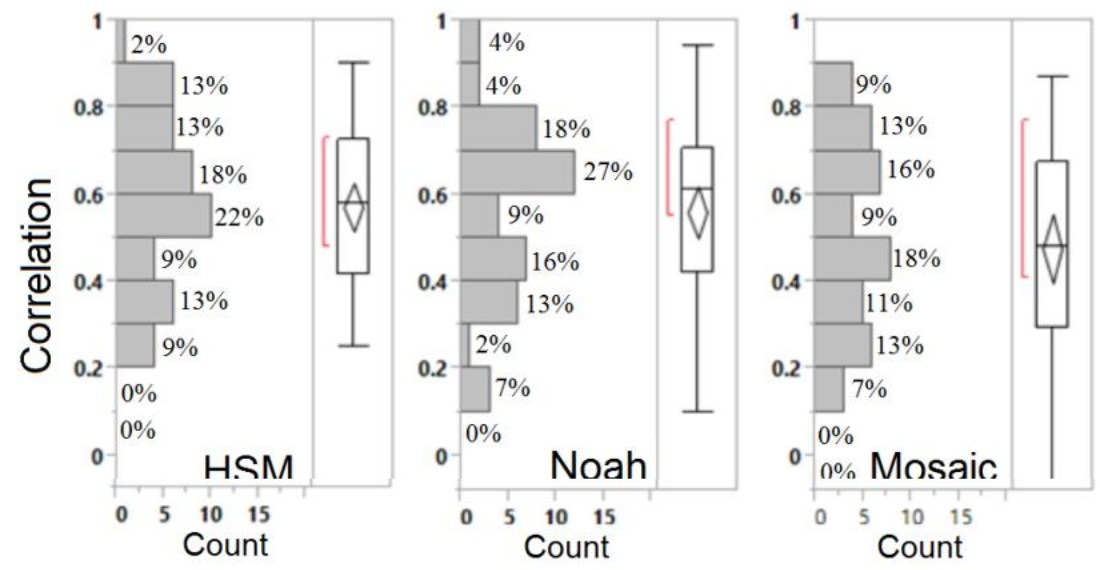

Fig. 8: Histograms for correlation of the observed in- situ soil moisture from control stations with HSM, Noah and Mosaic for the three layers. 
(a) In- situ data

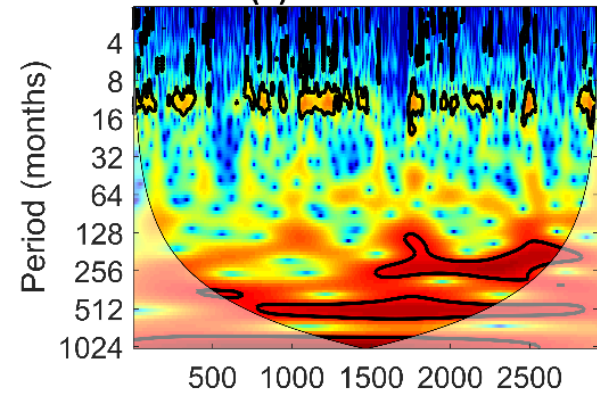

(c) Noah

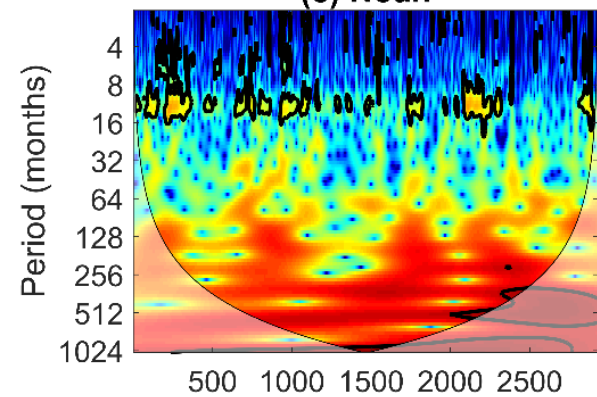

(b) HSM

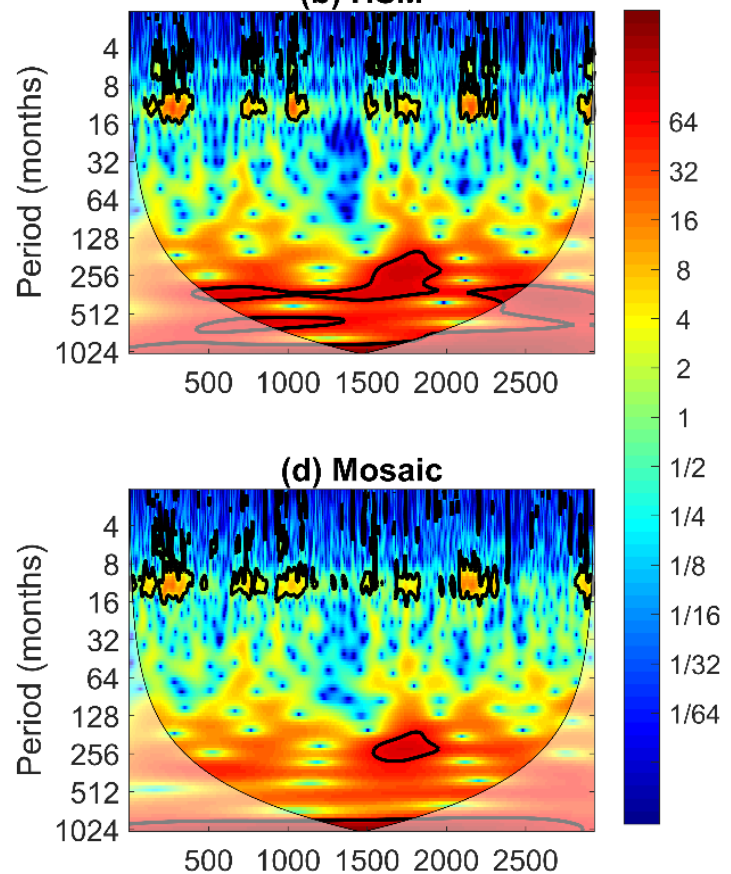

Fig. 9: Wavelet spectrums of (a) Observed in- situ soil moisture (b) Hybrid Soil Moisture (c) Noah (d) Mosaic time series from the control stations for total dataset for layer 1 using Morlet wavelet. The thick black contour delineates the 5\% significance level against the red noise. Red and blue colours represent stronger and weaker power respectively. 


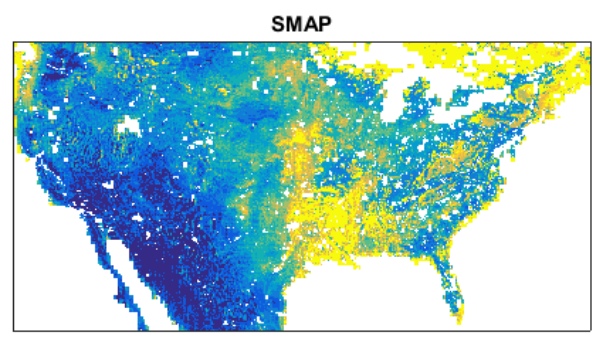

Noah

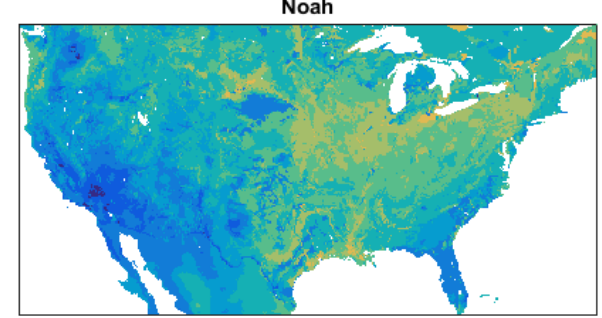

Volumetric soil moisture $\left(\mathrm{cm}^{\star \star} 3 / \mathrm{cm}^{\star \star} 3\right)$

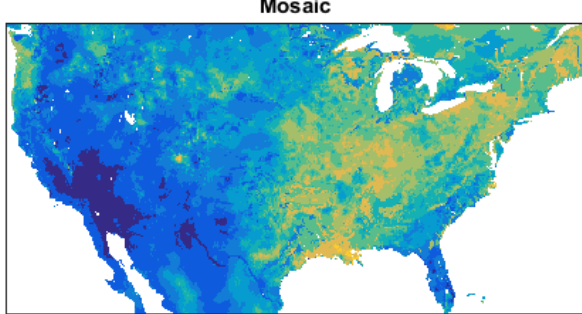

June 2015
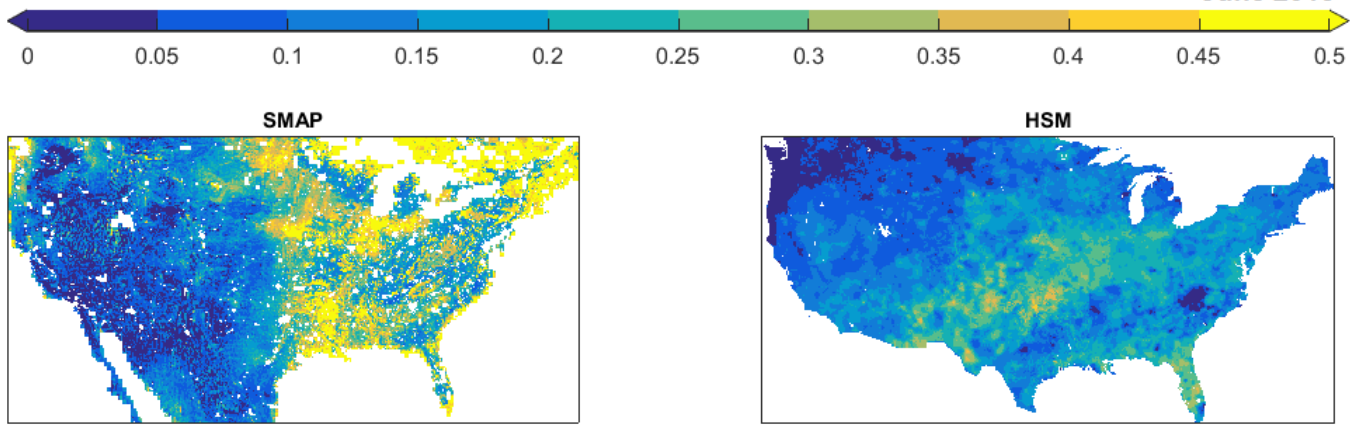

Noah

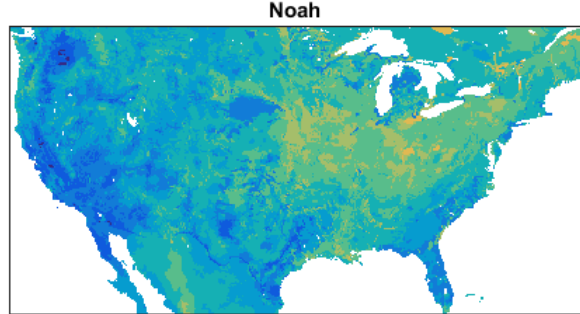

Volumetric soil moisture $\left(\mathrm{cm}^{\star \star} 3 / \mathrm{cm}^{\star \star} 3\right)$

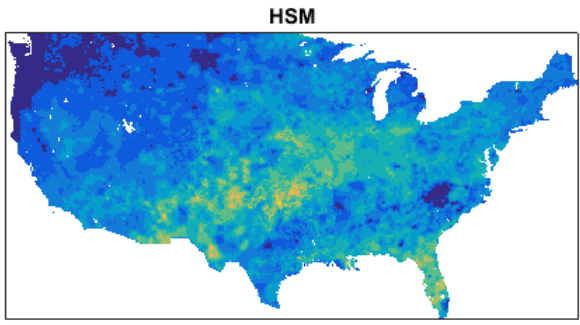

Mosaic

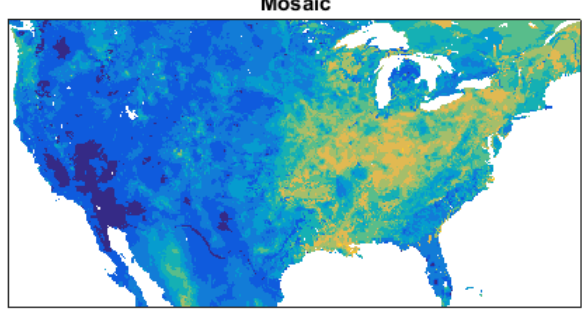

July 2015

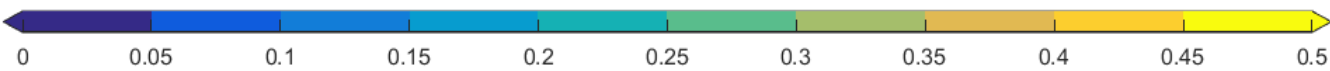

Fig. 10: Comparison of op soil layer estimation from SMAP, HSM, Noah and Mosaic for June and July 2015. 

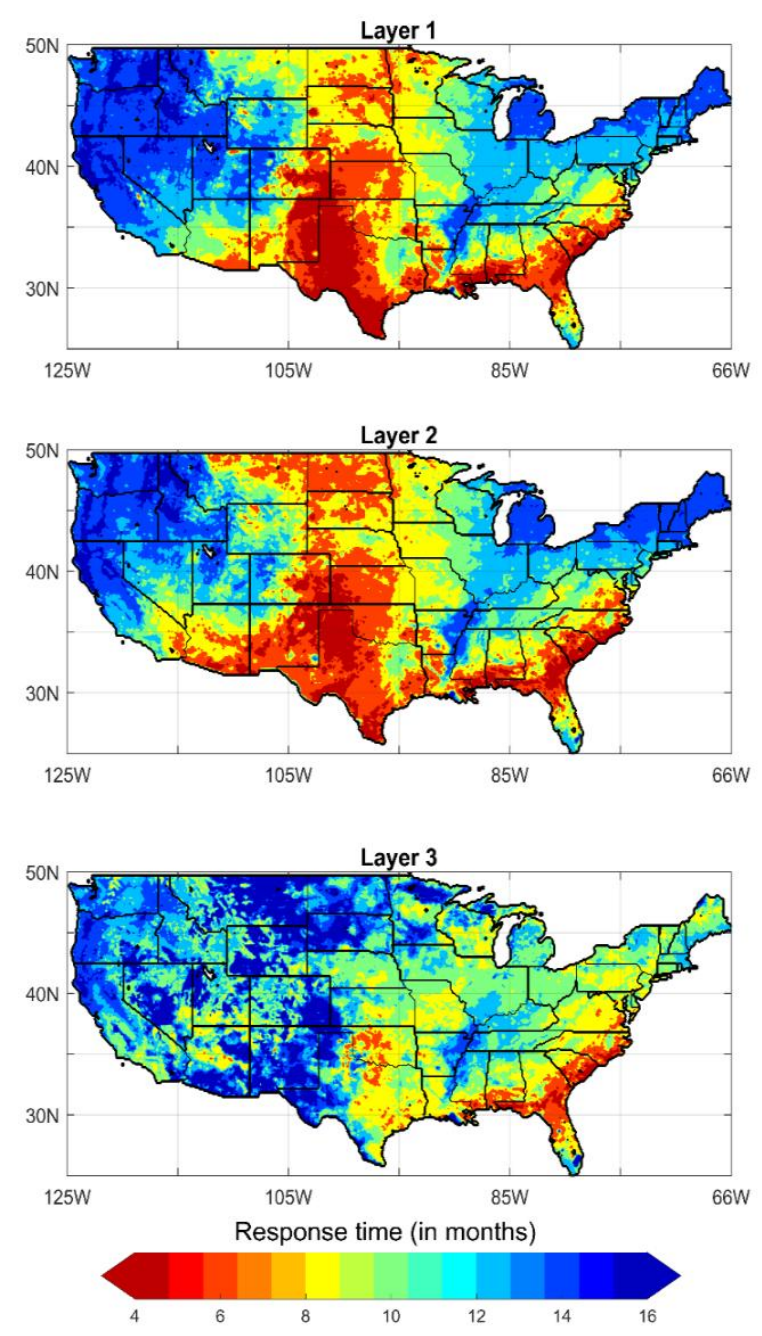

Fig. 11: Spatial distribution of Response Time $\left(T_{o}\right)$ of Hybrid Soil Moisture (in months) for the three layers. Red/ Orange represents low persistence whereas Blue/ Cyan represents regions with higher Persistence. 


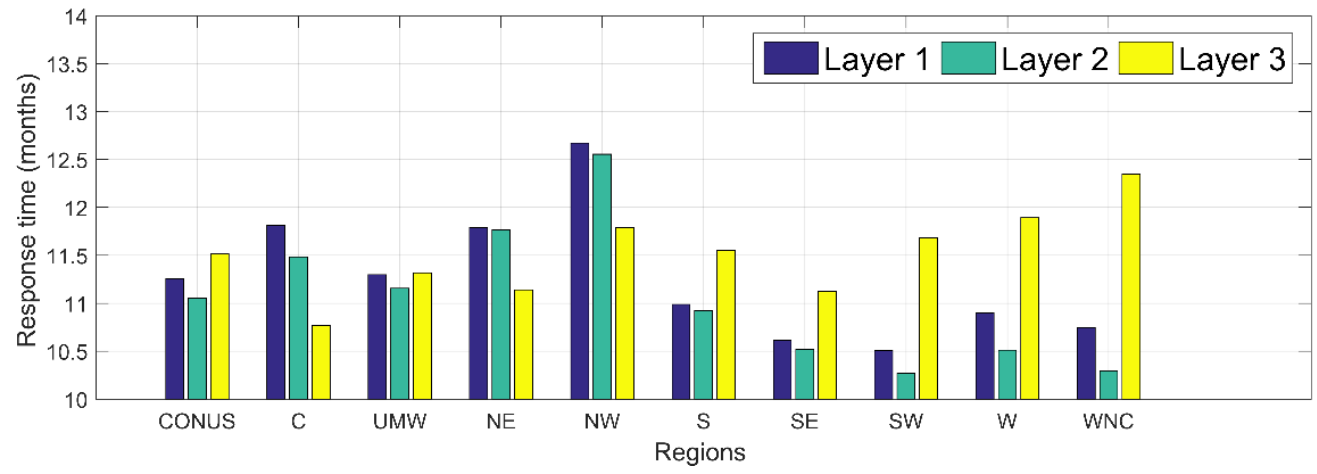

Fig. 12: Mean Response time $\left(T_{o}\right)$ for three soil layers for the nine climate regions of the US $(\mathrm{C}=$ Central, UMW=Upper Midwest, $\mathrm{NE}=$ North East, $\mathrm{NW}=$ North West, $\mathrm{S}=$ South, $\mathrm{SE}=$ South East, SW= South West, W= West, WNC= West North Central). 

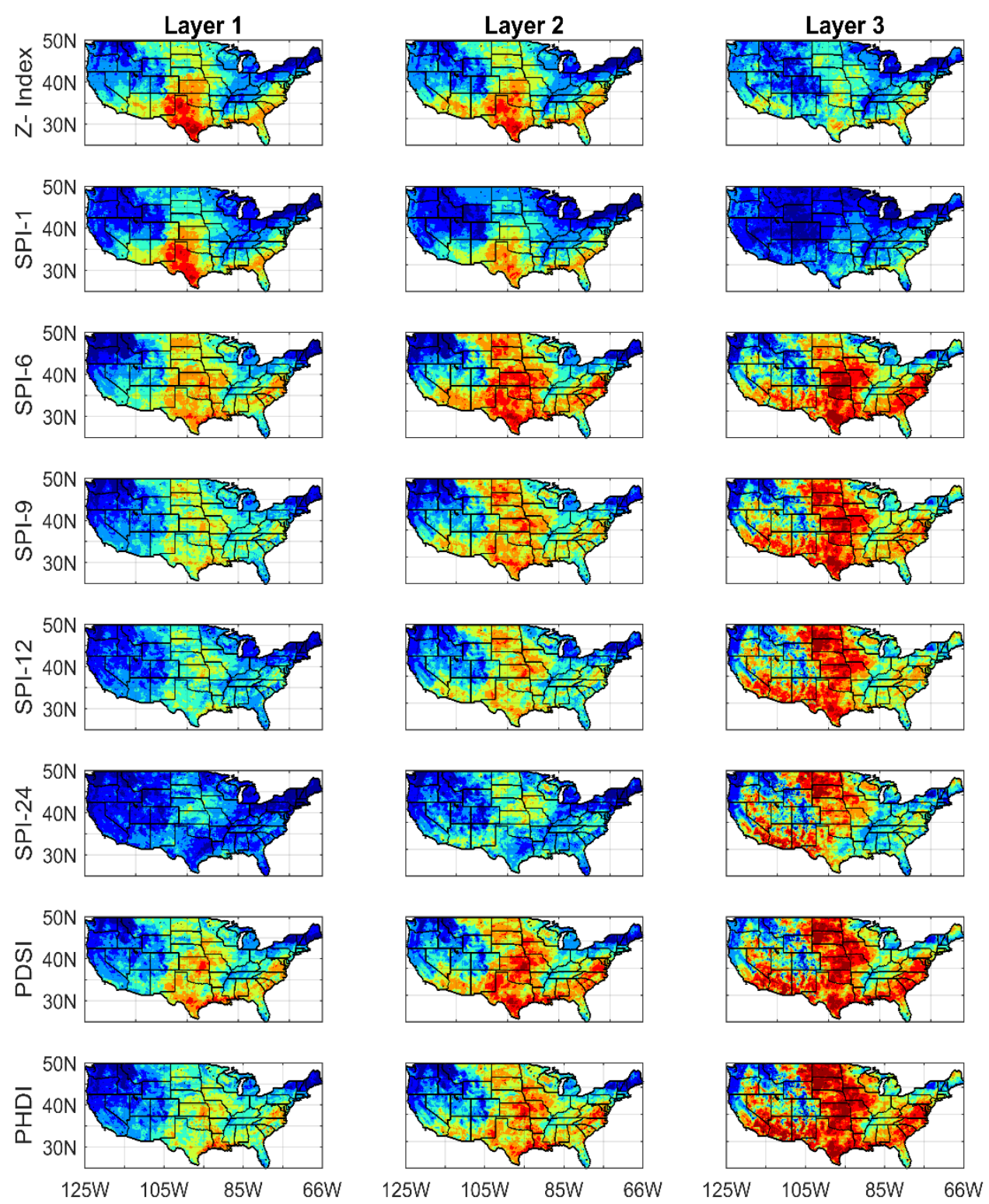

Correlation of HSM with drought indices

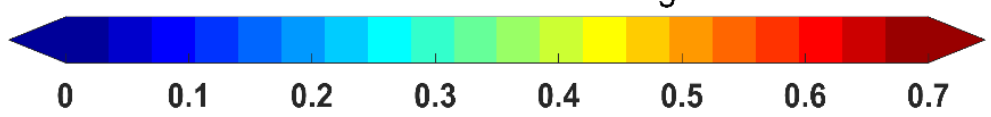

Fig. 13: Correlation plots of Hybrid Soil Moisture with the eight drought indices for the three soil layers for CONUS. Red color represents stronger positive correlation whereas Blue color represents near low or negative correlation with the drought indices. 

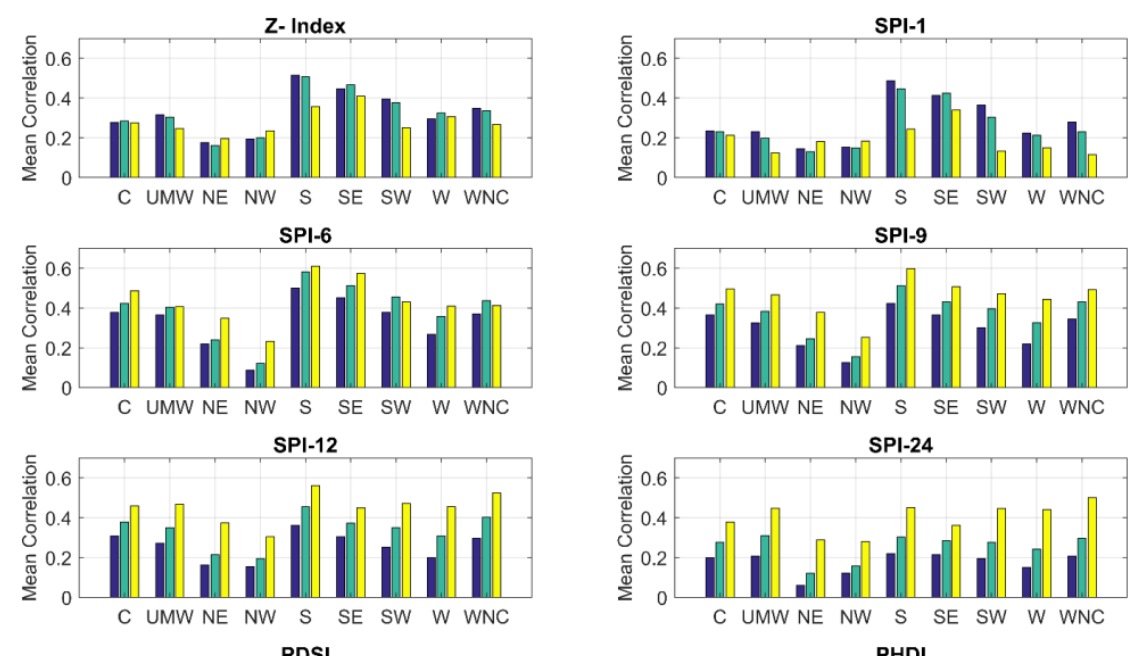

SPI-24
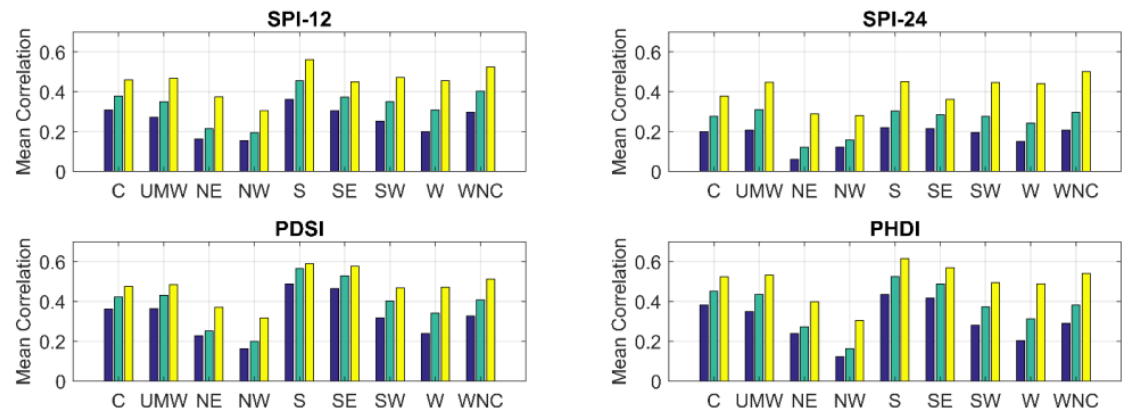

$\square$ Layer $1 \square$ Layer $2 \square$ Layer 3

Fig. 14: Mean correlation of soil moisture with drought indices (DIs) for the nine climate regions of the U.S. $(\mathrm{C}=$ Central, UMW=Upper Midwest, $\mathrm{NE}=$ North East, $\mathrm{NW}=$ North West, $\mathrm{S}=$ South, $\mathrm{SE}=$ South East, $\mathrm{SW}=$ South West, W= West, WNC= West North Central).
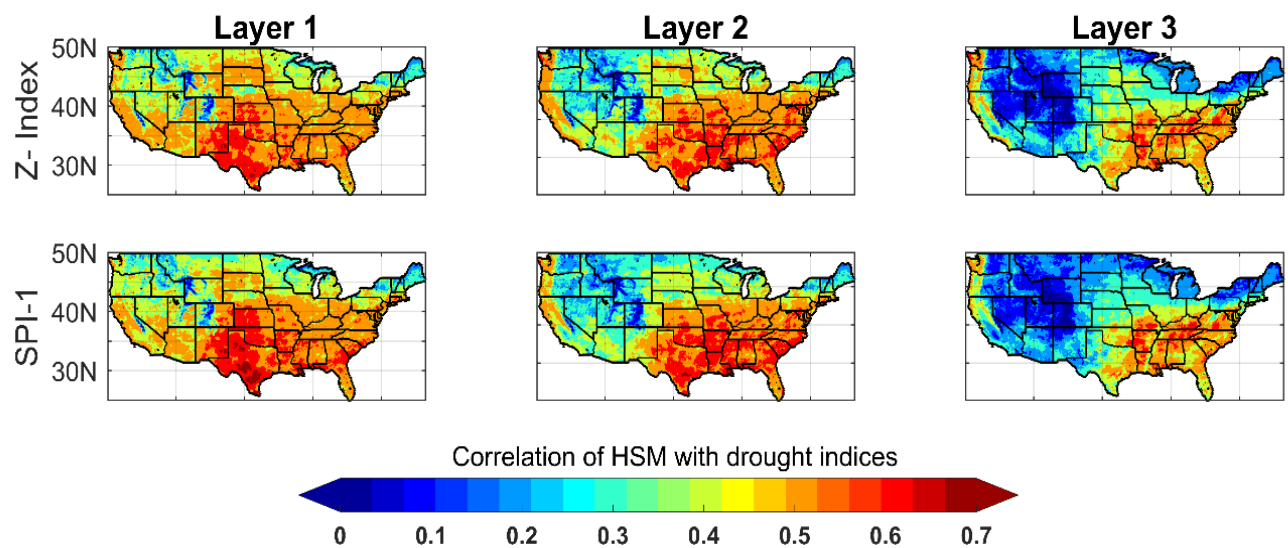

Fig. 15: Correlation plots of pre- whitened Hybrid Soil Moisture with pre- whitened Z- index (top row) and SPI-1 (bottom row) for the three soil layers for CONUS. Red color represents stronger positive correlation whereas Blue color represents near low or negative correlation with the drought indices. 


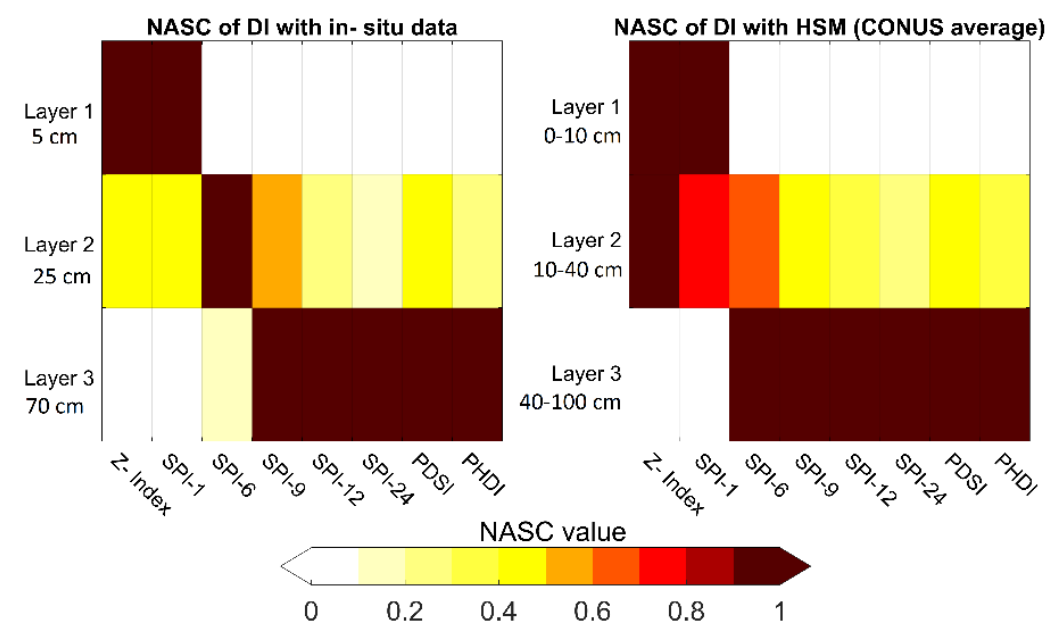

Fig. 16: The Averaged Squared Correlation normalized across three soil layers (Read top to bottom) to obtain the Normalized Averaged Squared Correlation (NASC) for both in- situ US Climate reference network (USCRN) data and Hybrid Soil Moisture averaged over CONUS. $\mathrm{NASC}=1$ indicates strongest correlation, $\mathrm{NASC}=0$ indicates weakest, and, $0<\mathrm{NASC}<1$ indicates intermediate correlation between the soil moisture of the respective layer and a given drought index. 


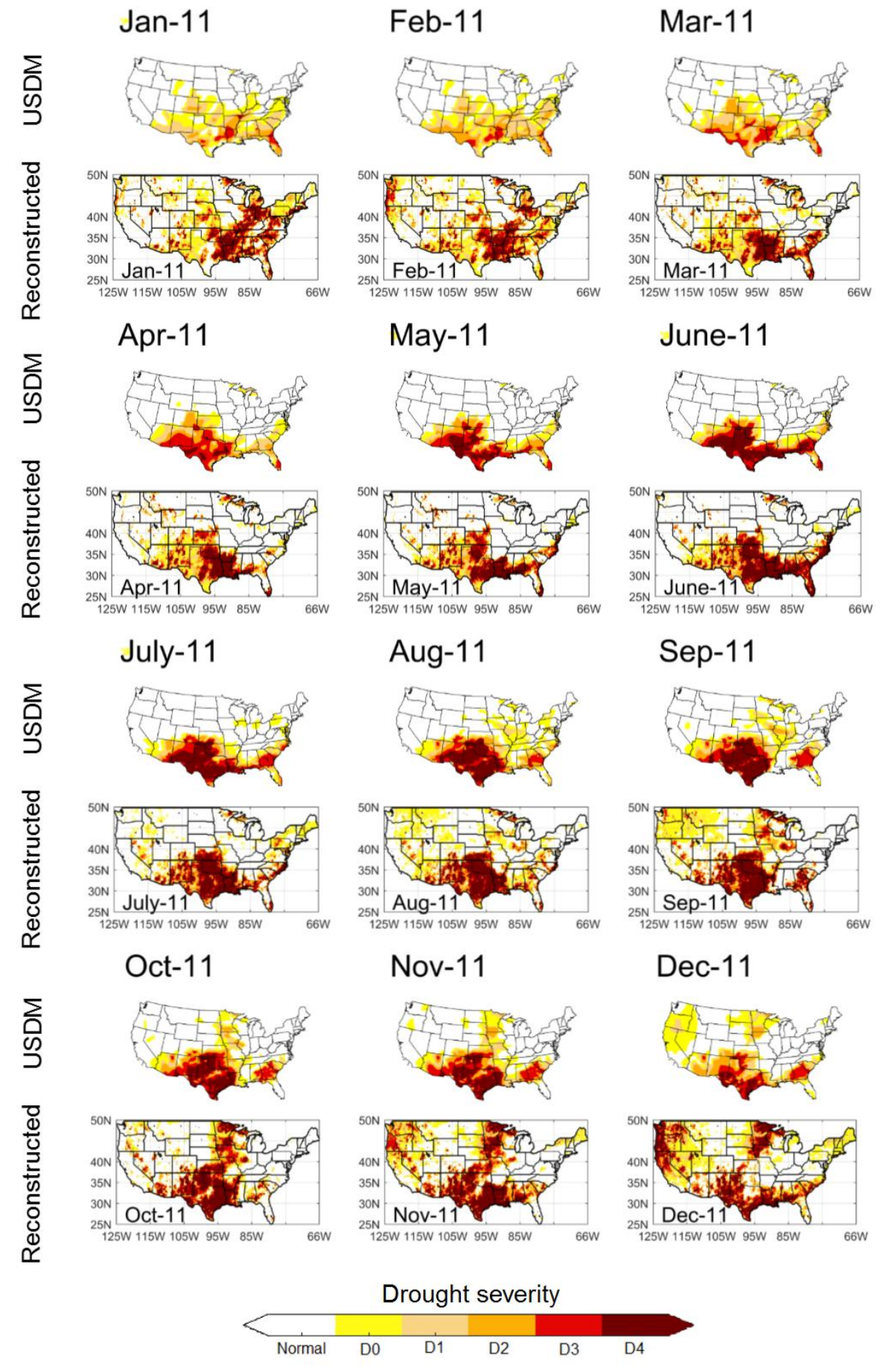

Fig. 17: Reconstructed drought maps using the proposed layer-wise soil moisture percentile approach using HSM v/s USDM drought maps for CONUS (Apr'-11 to Dec'- 11) 

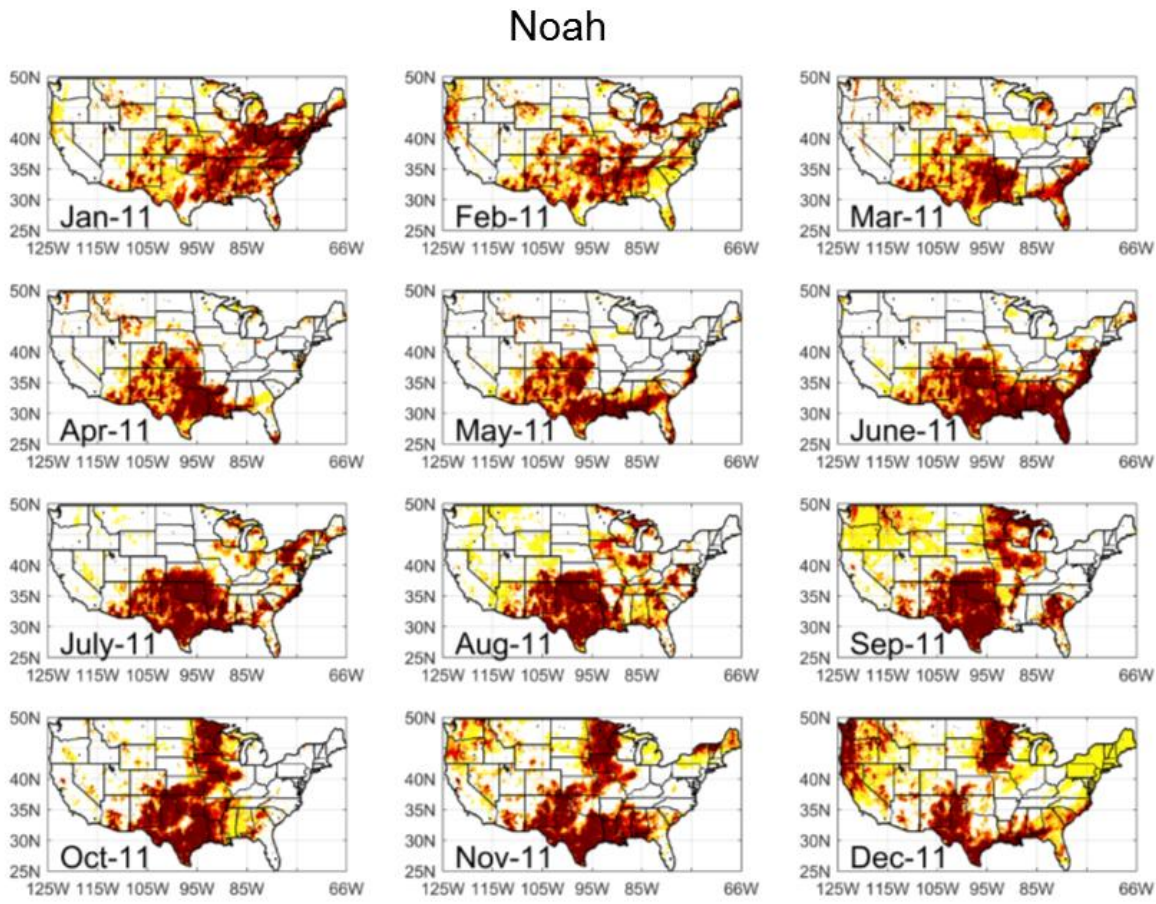

\section{Mosaic}
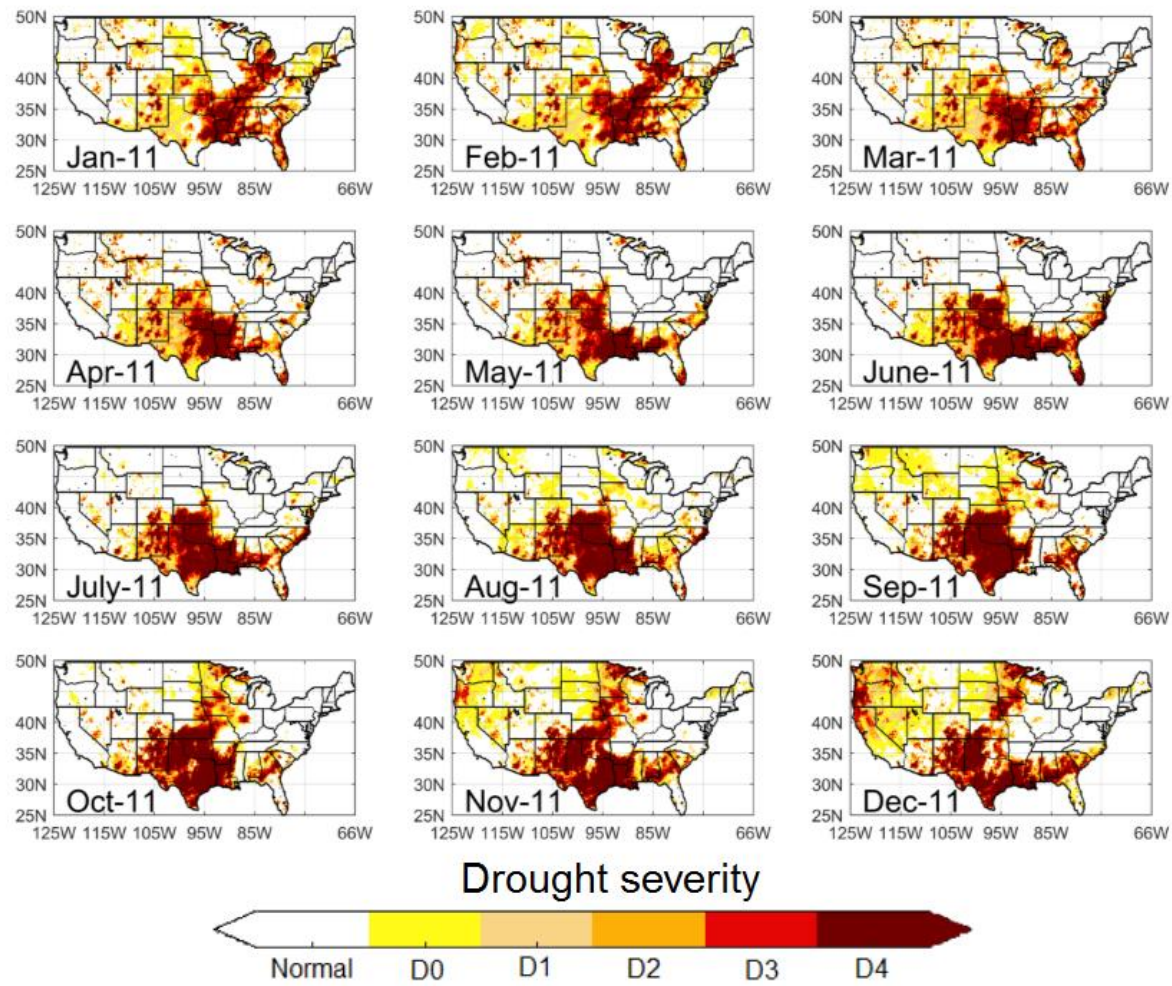

Fig. 18: Reconstructed drought maps using the proposed layer-wise soil moisture percentile approach using Noah and Mosaic dataset v/s USDM drought maps for CONUS (Apr'-11 to Dec'- 11) 
(a) HSM

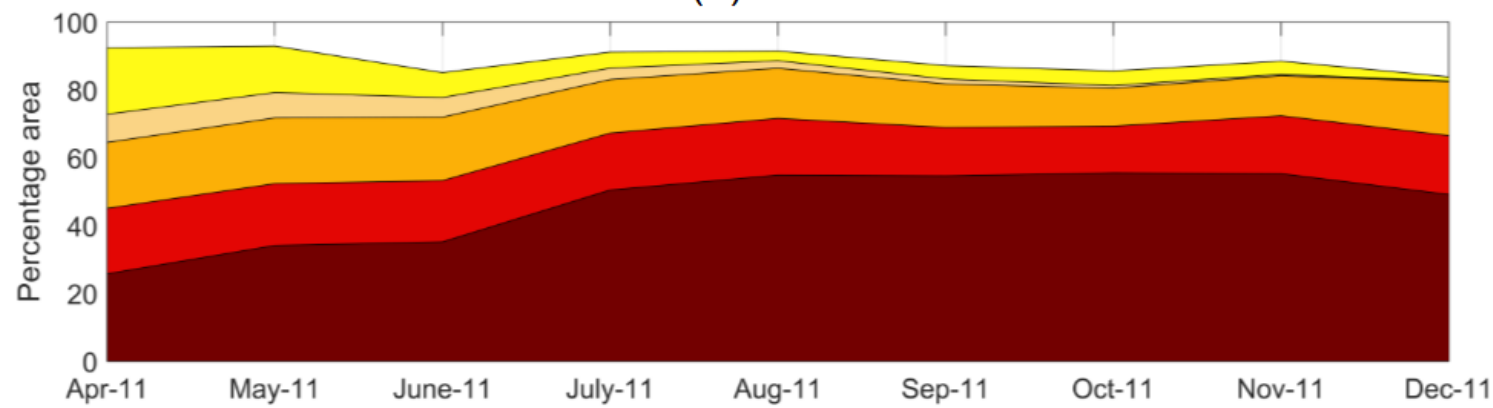

(b) Noah

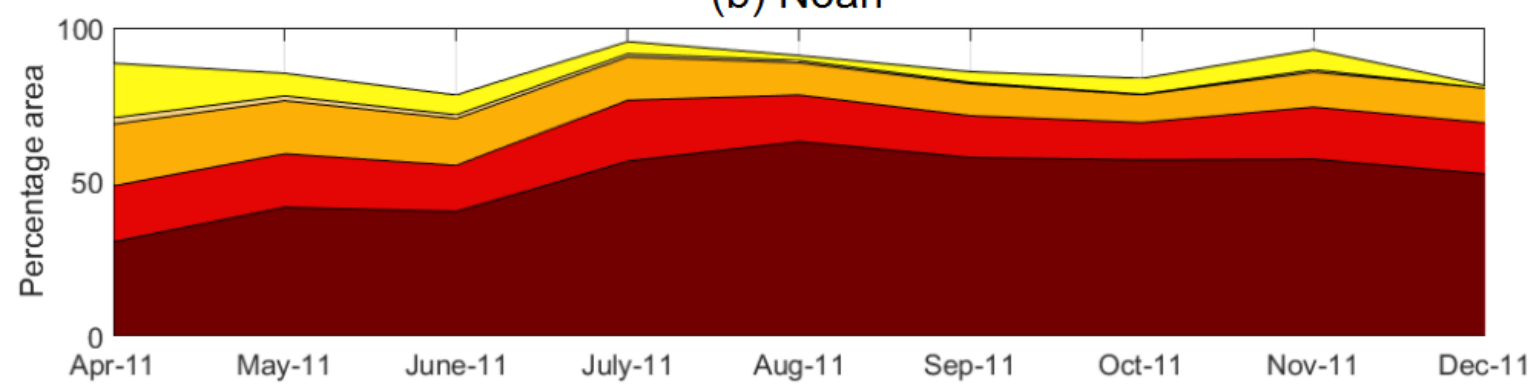

(c) Mosaic

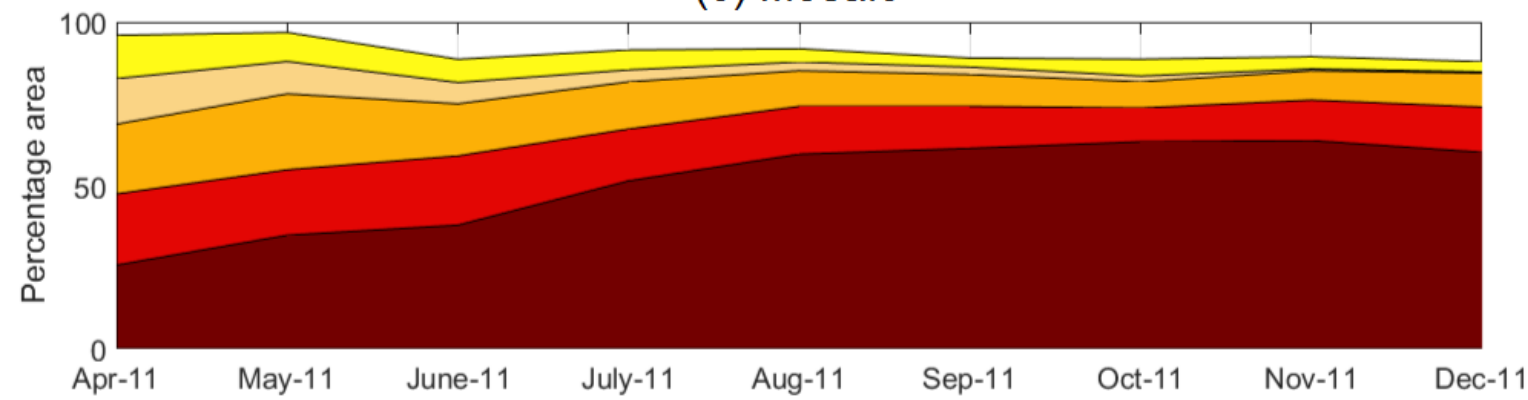

(d) USDM

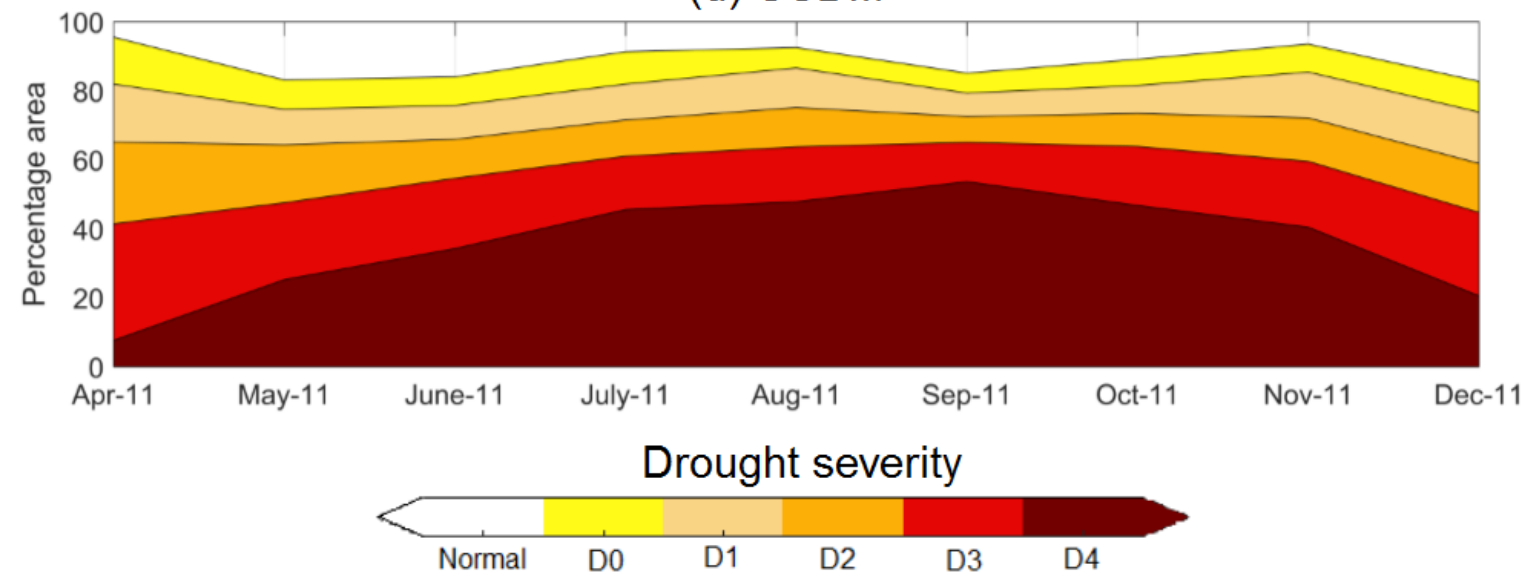

Fig. 19: Comparison of the area under various drought severities as obtained from the reconstructed drought maps using (a) HSM (b) Noah (c) Mosaic and (d) USDM maps (Apr'11 to Dec'- 11) for Southern U.S. 
(a)

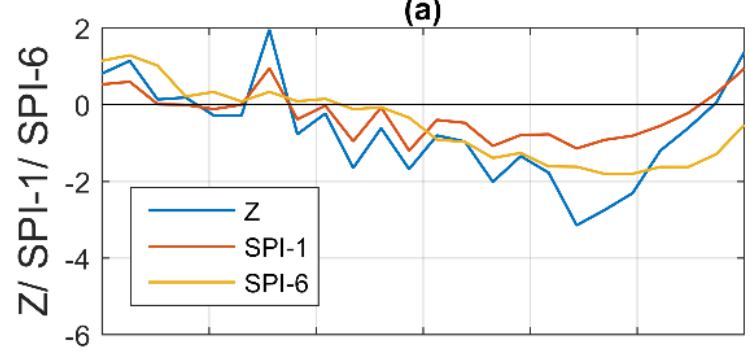

(b)

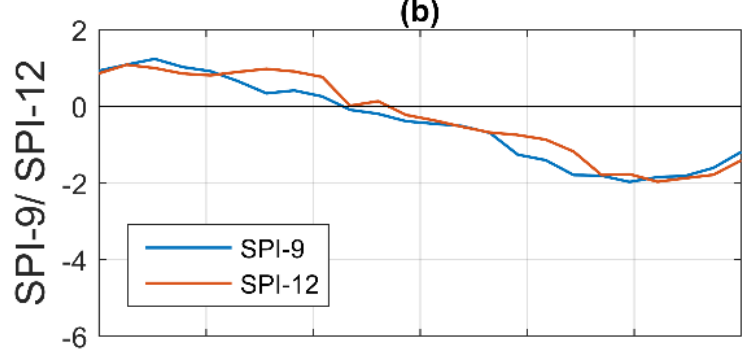

(c)

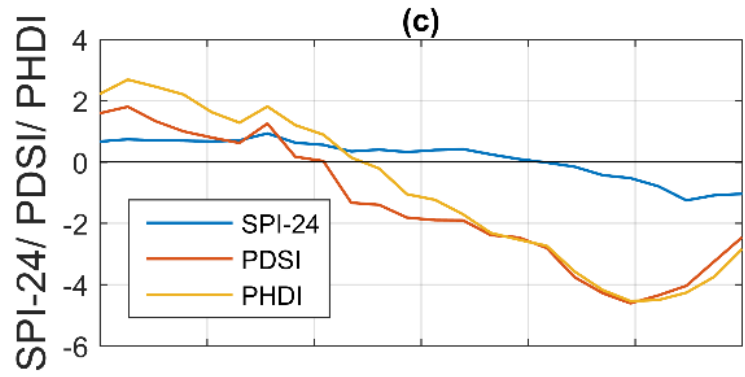

Jan-10 Apr-10 Aug-10 Dec-10 Apr-11 Aug-11 Dec-11

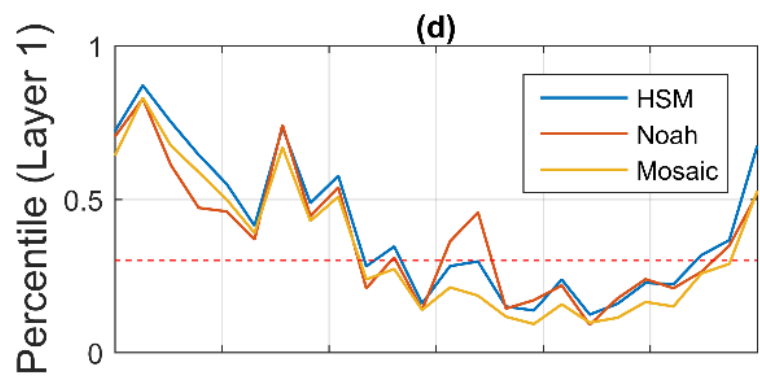

(e)

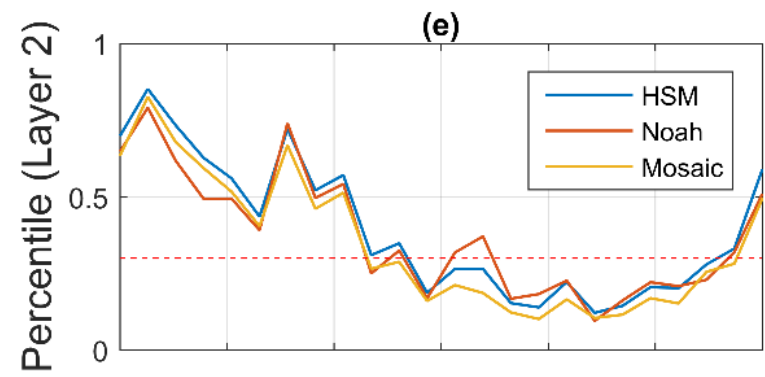

(f)

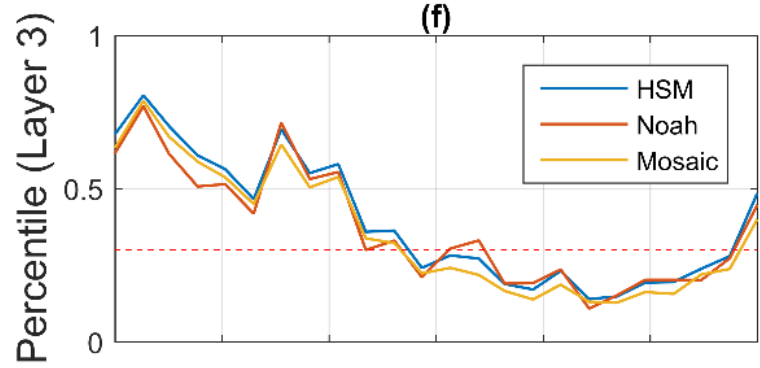

Jan-10 Apr-10 Aug-10 Dec-10 Apr-11 Aug-11 Dec-11

Fig. 20: Comparison of average value of the eight drought indices (Left) (a) Z-index, SPI-1 and SPI- 6 months (b) SPI- 9 and SPI- 12 (c) SPI- 24, PDSI and PHDI with monthly soil moisture percentiles obtained using HSM, Noah and Mosaic for (d) Layer 1 (b) Layer2 and (c) Layer 3 for Southern US. 